\title{
Action selection conflict and intentional binding: an ERP study
}

\author{
Roberta Vastano $^{\mathrm{a}, \mathrm{b}}$, Ettore Ambrosini ${ }^{1 \mathrm{c}, \mathrm{d}}$, José Luis Ulloa ${ }^{1 \mathrm{a}, \mathrm{e}}$, Marcel Brass ${ }^{\text {a }}$ \\ 1 equal contribution
}

a) Department of Experimental Psychology, Ghent University, Henri-Dunantlaan 2, 9000 Ghent, Belgium.

b) University of Miami, Department of Neurological Surgery, the Miami Project to Cure Paralysis, Miami, FL 33136

c) Department of General Psychology, University of Padua, Padua, Italy

d) Department of Neuroscience, University of Padua, Padua, Italy

e) Centro de Investigación en Ciencias Cognitivas, Facultad de Psicología, Universidad de Talca, CP 3460000, Chile

\section{Corresponding author:}

Roberta Vastano,

University of Miami, Department of Neurological Surgery, the Miami Project to Cure Paralysis, Miami, FL 33136

roberta.vastano@gmail.com

\section{Keywords:}

Intentional binding, action fluency, P300, pre-response central-positivity, post-response central negativity, threshold-free cluster-enhancement (TFCE) 


\section{Abstract}

The fluency with which we plan and execute actions has been demonstrated to increase our sense of agency (SoA). However, the exact mechanisms how fluency influences SoA are still poorly understood. It is an open question whether this effect is primarily driven by fluency of stimulus processing, response preparation or by processes following response execution. In the current study we aim at addressing this question by measuring event-related potentials reflecting pre- and post-response mechanisms and relate them to intentional binding, a measure of implicit SoA. To manipulate the fluency of action we asked participants to perform actions that were congruent or incongruent with a visual target (a finger movement). Participants' actions triggered an auditory outcome. To measure the intentional binding effect we asked participants to estimate the time between the executed actions and the ensuing auditory effects. We found that congruent actions generated a larger intentional binding effect (i.e. stronger time compression between actions and effects) and this positively correlated with a late $\mathrm{P} 300$ evoked during the processing of congruent stimuli. At the action selection level, we found a larger central pre-response positivity for incongruent condition as relates to interference effects. Finally, post response mechanisms elicited a larger central negativity for incongruent responses presumably related to uncertainty. We provide new evidence on the determinants of intentional binding driven by the fluency of action, by showing that both pre and post-response mechanisms are crucial in the generation of the feelings of agency. Importantly, stimulus processing and response preparation ERPs seem to be more selectively modulated by congruency-effects given specific brain-behavioral correlations. 


\section{Introduction}

Any action that we perform carries consequences. The feeling of control over action consequences is referred to as sense of agency (SoA) (David, Newen, \& Vogeley, 2008; Haggard \& Tsakiris, 2009). It has been shown that the SoA depends on the fluency of action, that is, the SoA arises prospectively, prior the action effects and it is boosted in situations where people act in accordance with their intentions, when no discrepancy occurs at the action selection level (Chambon \& Haggard, 2012; Chambon, Sidarus, \& Haggard, 2014; Sidarus \& Haggard, 2016). In a previous behavioral study (Vastano et al., 2017) we showed that an implicit measure of SoA, known as intentional binding effect (a perceived time compression between the voluntary action and its consequent effect) is modulated by action selection processes. We demonstrated that the congruency between observed supraliminal primes and executed actions (action-congruency effect) boosted the intentional binding effect, while a conscious conflict at the action selection level reduced it. Our previous results, therefore, indicated a tight relationship between action-congruency and the intentional binding. However, whether this relationship is primarily related to fluency in stimulus processing, response selection or processes following response execution is still poorly understood.

Although several studies have been conducted to identify electrophysiological correlates of the intentional binding effect (Gentsch \& Schütz-Bosbach, 2011; Goldberg, Busch, \& Meer, 2017; Hughes, Desantis, \& Waszak, 2013a; Jo, Wittmann, Hinterberger, \& Schmidt, 2014; Kühn et al., 2011), they primarily focused on the processing of action outcomes. For instance, studies that used electroencephalography (EEG) showed that inferential and predictive mechanisms during voluntary actions induce a suppression of the N100 over central electrodes for self-generated and congruently predicted as compared with externally-generated action outcomes (Gentsch \& Schütz-Bosbach, 2011; Hughes et al., 2013a; Hughes, Desantis, \& Waszak, 2013b; Poonian, Mcfadyen, Ogden, \& Cunnington, 2015). Similarly, another ERP response has been shown to depend on the contiguity of self-generated outcomes. The feedback-Correct Related Positivity (fCRP) is a positive-going ERP response elicited to positive/correct outcomes around $250 \mathrm{~ms}$ after the stimulus onset over frontal-central electrodes. This fCRP is larger for self-generated as compared to externally-generated feedbacks (Bednark \& Franz, 2014).

Beyond this focus on the processing of action outcomes, a recent EEG study investigated the effect of action preparation on the SoA. In this study explicit judgments of agency were modulated using subliminal action-primes (Sidarus, Vuorre, \& Haggard, 2017). The authors showed that an unconscious influence, for instance the incompatibility between subliminal primes and performed actions reduced the explicit SoA. This behavioral effect was associated with early ( $100 \mathrm{~ms})$ 
negative ERPs such as the correct related negativity (CRN), that it has been interpreted as (Grützmann, Riesel, Klawohn, Kathmann, \& Endrass, 2014; Kwapil, Barrantes-Vidal, \& Silvia, 2008; Pailing \& Segalowitz, 2004). However, other types of stimuli, like supraliminal primes, might exert strong conflict effects during action selection and lead to different ERPs modulations associated with stimulus processing and preparatory action phase. To date, there is no evidence of neural correlates associated with the impact of a conscious conflict during action preparation and execution on the intentional binding.

In the present study, we investigate whether intentional binding is primarily driven by fluency induced by stimulus processing and response preparation or rather by processes following response execution. We are interested in elucidating how pre- and post-action processes, rather than processing of action outcomes, influence the intentional binding. Our aim was, therefore, to investigate the neural correlates of the fluency of action (manipulating the action-congruency effect) on the intentional binding by analyzing the perceived influence of a supraliminal actionprime that would facilitate or interfere with action selection processes. To this end we used an imitation-inhibition task, a type of ideomotor compatibility paradigm (Brass, Bekkering, Wohlschläger, \& Prinz, 2000; Brass, Bekkering, \& Prinz, 2001; Brass, Derrfuss, \& von Cramon, 2005). According to the ideomotor theory (Greenwald, 1972) the high similarity between the observed action and the required one induces a strong response tendency, and so the observed stimulus strongly overlaps with the anticipated effect of the planned action (see also the dimensional overlap model by (Kornblum, Hasbroucq, \& Osman, 1990). In the context of our study, the direct activation of a motor representation should have a strong impact on agency. This might be reflected in changes at the level of anticipations of the effect and changes in motor preparation.

We recorded EEG signals while participants performed the imitation-inhibition task in combination with a time estimation task (which allows us to measure intentional binding). Participants were instructed to lift their index or middle finger in response to a number, while simultaneously observing either congruent or incongruent finger movements of a mirrored righthand. Their action caused an auditory effect after a variable interval. At the end of each trial, participants estimated the time between their action and the ensuing effect. We computed stimulus-locked ERPs associated with stimulus processing and response-locked ERPs associated with motor preparation and post response action monitoring. In order to fully disclose the spatiotemporal dynamics of the fluency of action on intentional binding we used a data-driven approach. We investigated differences in the entire spatio-temporal space with the threshold-free cluster- 
enhancement technique (see method section). Finally, to investigate the relationship between the related behavioral effects (intentional binding measures).

We predict a larger intentional binding effect for the congruent compared with the incongruent and baseline conditions, as previously showed (Vastano et al., 2017). At the neural level, we expect to find modulation of ERPs as a function of the action-congruency effect. One likely candidate is the P300 component. Previous studies have provided evidence of a larger P300 amplitude for the congruent (where the observed action is consistent with the intended one) relative to the incongruent condition (Deschrijver, Wiersema, \& Brass, 2017). In addition, it has been also shown that the P300 is larger for congruent compared with incongruent conditions in other stimulus-response compatibility paradigms (Polich, 2007; Ragot \& Fiori, 1994; Sebanz, Knoblich, Prinz, \& Wascher, 2006; Zhou, Zhang, Li, Tan, \& Han, 2004) due to a higher cognitive load in incongruent conditions reducing attentional resources needed for generating the P300 component.

On the basis of this previous evidence we expect larger P300 amplitude for the congruent relative to the incongruent condition. In addition, we expect to find a positive correlation between the P300 and the intentional binding effect for the congruent condition (when the observed target is consistent with the motor plan). This might provide evidence of a tight relationship between the P300 modulation and implicit SoA during stimulus processing and pre-response mechanisms. We also expect to find modulations of ERPs evoked during the response period. Previous studies on motor conflict have shown fronto-central negativities both during the pre-response (e.g Readiness Potential) and post-response (e.g. CRN) periods. These responses have been associated with motor preparation (Jo et al., 2014; Libet, Gleason, Wright, \& Pearl, 1983; Shibasaki \& Hallett, 2006; Waszak et al., 2005) and decision uncertainty (Grützmann et al., 2014; Kwapil et al., 2008; Pailing \& Segalowitz, 2004), respectively. On the basis of these previous studies we expect that the conscious conflict at the action selection level (such as in the incongruent condition) will induce larger pre- and post-response fronto-central negativities relative to when conflict is not present (congruent and baseline conditions). We finally predict that pre-response rather than post-action ERP components would be strongly related to intentional binding, given the pivotal role of the fluency at early action selection level on the SoA (Chambon \& Haggard, 2012).

\section{Materials and Method}

\subsection{Participants}


We report how we determined our sample size, all data exclusions (if any), all inclusion/exclusion criteria, whether inclusion/exclusion criteria were established prior to data analysis, all manipulations, and all measures in the study.

Twenty-eight healthy volunteers (8 males, mean age: 23.25 years, SD: 2.5 years) were enrolled for the study. An a-priori sensitivity power analysis (G*Power 3 software; (Faul, Erdfelder, Lang, \& Buchner, 2007) revealed that our sample size is large enough to detect a significant behavioral effect for the Congruency factor (Vastano et al. 2017) in a repeated-measure ANOVA (rmANOVA) corresponding to either a large effect size $\mathrm{f}=0.4$ (Cohen, 1988), with a statistical power $(1-\beta)$ of .95 or a medium effect size $f=0.25$ with a statistical power of .8 (given $\alpha=$ .05 , a correlation between repeated measures of 0.5 , number of groups $=1$, number of measurements $=3$ ). All participants were right-handed, with normal or corrected to normal vision and no history of psychiatric or neurological disorders. All testing procedures were approved by local ethical committee of Ghent University and conducted in accordance with the Declaration of Helsinki. All participants gave written informed consent and were financially compensated for their participation.

\subsection{Stimuli and Procedure}

We used the same stimuli and procedure as in our previous work (Vastano et al. 2017). Participants were seated at a distance of $60 \mathrm{~cm}$ from a 24" computer monitor (resolution: $1920 \times$ 1080; refresh rate: $60 \mathrm{~Hz})$. Experimental stimuli consisted of a sequence of images $(300 \times 200$ pixels) of a mirrored right-hand of an actor performing lifting finger movements. The first image showed the hand in resting position for $1000 \mathrm{~ms}$ and was followed by two images lasting 16.67 $\mathrm{ms}$ in which the finger lifting movements (index or middle finger) and a number (1 or 2 that appeared between the two fingers) were shown in parallel. The last image of the sequence showed the finger in the end position, which stayed on the screen until the generated action effect (a pure tone at $1000 \mathrm{~Hz}$ ). The finger movements could result in a match or in a mismatch with the number showed (1: index finger and 2: middle finger in congruent trials; or 1: middle finger and 2: index finger in incongruent trials), or just the number (no movements) was shown (1 or 2 in baseline trials). At beginning of each trial a sentence "place your fingers" appeared on the screen and the participants were instructed to position their right index and middle finger on the " $\mathrm{G}$ " and " $\mathrm{H}$ " keys of a Mac keyboard with numeric keypad (MB110Z/B) by holding down the buttons. After a fixation cross (of a duration randomly varying between 1000 and $1600 \mathrm{~ms}$ in steps of $200 \mathrm{~ms}$ ) the image sequence started, and the participants had to lift their index or middle finger as fast as possible in response to the number (1: index finger and 2: middle finger) while observing 
congruent or incongruent fingers movements (Fig. 1). If no response was given within a time window of $1400 \mathrm{~ms}$, the next trial was presented, and the missed trial was recovered at the end of the experimental block. After their key release and a variable interval (300, 400, or $500 \mathrm{~ms})$ the auditory stimulus was delivered for $300 \mathrm{~ms}$ by means of headphones. After hearing the auditory stimulus (with a slight variable interval between 300 and 800 ms), a Visual Analogue Scale (VAS) appeared and we asked participants to estimate the interval between their action (key release) and the ensuing auditory effect ${ }^{1}$, by positioning the mouse pointer along the VAS from $100 \mathrm{~ms}$ to 900 ms (with markers indicating 200-ms intervals), they had a maximum of $5000 \mathrm{~ms}$ to give the answer. Finally, after a variable inter-trial interval ranging from 1000 to $3000 \mathrm{~ms}$ (in steps of 1000 ms) next trial started. Participants were told that the interval between their action and the tone was always chosen randomly in the range between $100 \mathrm{~ms}$ and $900 \mathrm{~ms}$. The experiment consisted of 240 randomized trials: 80 trials for each condition (congruent, incongruent and baseline), each of which was composed by $26-28$ trials for each interval $(300,400$, and $500 \mathrm{~ms})$. The experiment was divided in 4 little blocks of 60 trials each (20 trials in each congruent, incongruent and baseline) to allow participants to rest between blocks. Before the experiment there was a short training phase (21 trials). Furthermore, participants were trained to discriminate between 100 or $900 \mathrm{~ms}$ (the endpoints of the VAS scale used in the interval-time estimation task). They listened two tones separated by 100 or $900 \mathrm{~ms}$, at the end of each trial we asked them to indicate if the time elapsed between the two tones were 100 or $900 \mathrm{~ms}$. They received a feedback (correct or incorrect response). A number of 30 randomized trials (15 for each interval) were presented with a variable inter-trial interval ranging from 1500 to $2500 \mathrm{~ms}$. The task was implemented in E-prime 2.0 Professional software (Psychology Software Tools, Pittsburgh, PA). The duration of the whole experiment was about $80 \mathrm{~min}$. The stimuli we used and the script to run the experimental task are available from our project repository on the Open Science Framework (https://osf.io/49t23).

<Fig.1 here> Figure 1. Experimental paradigm. Time line of the trials. Participants responded according to a number ( 1 or 2 ) presented between the index and middle finger. They were instructed to lift the index finger if the number was 1 and the middle finger if the number was 2. After their movement with a random interval (300, 400 or $500 \mathrm{~ms}$ ) followed a tone (action outcome). At the end of each trial participants judged on a visual scale the time elapsed between their action and the tone. Congruent trials are the ones where the number corresponded with the

\footnotetext{
${ }^{1}$ We used a time estimation task widely used in several studies to measure the intentional binding effect (Caspar, Cleeremans, \& Haggard, 2015; Cravo, Claessens, \& Baldo, 2009; Engbert, Wohlschläger, Thomas, \& Haggard, 2007; Kühn, Brass, \& Haggard, 2013; Wen, Yamashita, \& Asama, 2015) because of the impossibility to combine the classical procedure which involves the Libet clock (Haggard, Clark, \& Kalogeras, 2002) with our task which requires to attend visual stimuli. In fact, the Libet clock procedure requires participants to watch a clock face with a clock-hand rotating and to react at the time that they chose. Therefore, it would have been impossible to ask participants to attend to the clock hand rotating and to react to the visual stimuli at the same time.
} 
background movement, Incongruent trials are the ones where number and movment did not match and Baseline trials are represented by numbers only.

\subsection{Electrophysiological Recording and Preprocessing}

EEG data were recorded from 64 electrodes placed according to the extended 10-20 EEG-system using an elastic cap and the Biosemi ActiveTwo system. To measure vertical and horizontal eye movements, bipolar electrodes were placed above and below the left eye, and on the outer canthi of both eyes. EEG signals were referenced online to the CMS-DRL ground and sampled at 1.024 Hz. Electrode offsets were kept between -25 and $25 \mu \mathrm{V}$ at all electrodes.

EEG signals were band-pass filtered between .25 and $45 \mathrm{~Hz}$ and processed and analyzed by using MATLAB-based custom scripts and EEGLAB toolbox (Delorme, Fernsler, Serby, \& Makeig, 2006). An automatic rejection of noisy EEG channels was performed on continuous data and confirmed by visual inspection. The channel rejection procedure was performed by using the "clean_channels" function in EEGLAB, which removed channels that shared less than $50 \%$ of the variance with their robust estimate (computed on the basis of a 100-points random sample consensus procedure on 16 surrounding channels) for more than $33.3 \%$ of the total recording time). The resulting contaminated channels were interpolated using spherical splines (Perrin, Pernier, Bertrand, \& Echallier, 1989) . Overall, only five electrodes (F6, FC6, T7, P2, and Iz) were interpolated across eight different participants. Continuous EEG data were then re-referenced offline to the average of all of the electrodes and segmented into epochs of $3 \mathrm{~s}(0.5 \mathrm{~s}$ before the appearance of the first image). We included in the analyses only trials with correct behavioral responses (imitation-inhibition task). Successively, stereotypical artifacts, such as blinks, heartbeat, eye movements and muscle tension, were removed by Independent Component Analysis (ICA).

Finally we segmented stimulus-locked epochs between - 200 to $500 \mathrm{~ms}$ with a baseline correction of $200 \mathrm{~ms}$ before the stimulus onset (Target epochs) as well as response-locked epochs between 600 to $300 \mathrm{~ms}$ with a baseline correction of $200 \mathrm{~ms}$ (-600 to $-400 \mathrm{~ms}$ before response onset) (Preresponse epochs), and between -100 to 500 with a baseline correction of $100 \mathrm{~ms}$ (Post-response epochs). We then performed an automatic detection and rejection of artifactual and/or outlier EEG data epochs by applying different methods (see (Delorme \& Makeig, 2004), based on criteria that were determined through preliminary inspections and tests aimed at optimizing artifact rejection in our sample: $\pm 100 \mu \mathrm{V}$ for the standard extreme values thresholding; current drifts $>50 \mu \mathrm{V}$ with a correlation $>0.6$ for the linear trend test; SD $>7$ and 3 (across epochs and channels, respectively) 
for the improbability and kurtosis tests. Epochs violating any of these criteria were excluded from further analyses. Three separate grand average waveforms (Baseline, Congruent and Incongruent) for each Target, Pre-response and Post-response epoch were then constructed. The mean number of trials per participant (with ranges in parentheses) that contributed to each grand average was: Target (Baseline: 65 (38-78), Congruent: 65 (38-77), Incongruent: 57 (36-75)); Pre-response (Baseline: 63 (35-79), Congruent: 65 (39-76), Incongruent: 56 (36-73)); Post-response (Baseline: 64 (37-78), Congruent: 64 (38-77), Incongruent: 56 (36-73)). The raw data of our participants (with anonymous ID) and the scripts for EEG analysis are available from our project repository on the Open Science Framework (https://osf.io/49t23).

\subsection{Statistical analysis}

The behavioral and ERP datasets for statistical analysis are available from our project repository on the Open Science Framework (https://osf.io/49t23). We performed one-way repeated measure analysis of variance (rmANOVA) on the time-judgment errors (i.e., the difference between the estimated intervals and the actual intervals), with Congruency (3 levels: baseline, congruent and incongruent) as factor. For each participant and condition, observations more than 2 SDs away from the mean were excluded from the analysis. Additional one-way rmANOVAs was conducted on RTs and Error rates in the imitation-inhibition task with Congruency ( 3 levels: baseline, congruent and incongruent) as factor. Significant effects found in the rmANOVA were followed by Newman-Keuls-corrected post hoc tests. Alpha level was fixed at 0.05 for all statistical tests.

For each epoch of analysis, differences in ERPs across experimental conditions (Baseline, Congruent, Incongruent) were tested for statistical significance using a mass-univariate approach based on the threshold-free cluster-enhancement (TFCE) method, which optimizes the detection of both diffuse, low amplitude effects and localized, high amplitude ones while correcting for multiple comparisons with non-parametric permutation tests (Smith \& Nichols, 2009). This method was applied by taking into account all the 64 channels and all the time points composing the epochs of interest. In brief, for each time point and channel, an rmANOVA was performed, yielding (in the case of the Target epoch) $\approx 46,000 \mathrm{~F}$ values ( 717 time points * 64 channels). These $\mathrm{F}$ values were then TFCE-transformed and corrected for multiple comparisons with permutation tests (2500 resampling). Post-hoc pairwise t-tests were also performed with the same approach to clarify the pattern of differences for significant effects.

TFCE-corrected mass-univariate brain-behavior correlation analyses were also performed between the participants' raw pairwise differences in ERPs (Incongruent-Congruent, Congruent- 
Baseline, Incongruent-Baseline) for each time-channel data point and the same differences in the limit the description of the significant correlations to those involving ERP effect that were significant at the mass univariate ERP analyses, that is, to the ERP components that were both significantly modulated by the experimental conditions and, in turn, significantly modulated participants' behavioral performance (intentional binding measures); in other words, we will describe the results of intersection analyses between the mass-univariate ERP significant results and the mass-univariate brain-behavior significant correlations. Moreover, we will not describe significant correlations that involve less than ten consecutive time-points (i.e., significant effects lasting $<10 \mathrm{~ms}$ ) and less than three contiguous channels.

\section{No part of the study procedures and analysis was pre-registered prior to the research being conducted.}

3. Results

3.1 Behavioral results

\section{Imitation-inhibition task}

The ANOVA on Error rates revealed a significant effect of Congruency $\left(\mathrm{F}_{(2,54)}=35.53 ; \mathrm{p}<0.001\right.$; $\eta 2 \mathrm{p}=0.57$ ), with more errors in incongruent condition (Mean $=12.5 \%, \mathrm{SD}=10 \%$ ) as compared to congruent (Mean $=1.6 \%, \mathrm{SD}=2.2 \%)$ and baseline (Mean $=2.9 \%, \mathrm{SD}=2.5 \%)$ conditions (all ps $<0.001)$.

The ANOVA on RTs revealed a significant effect of Congruency $\left(\mathrm{F}_{(2,54)}=108.93 ; \mathrm{p}<0.001 ; \eta 2 \mathrm{p}\right.$ $=0.80$ ), with faster RTs in the congruent condition (Mean $=427 \mathrm{~ms} ; \mathrm{SD}=63 \mathrm{~ms}$ ) than incongruent (Mean $=493 \mathrm{~ms} ; \mathrm{SD}=78 \mathrm{~ms}$ ) and baseline (Mean $=475 \mathrm{~ms} ; \mathrm{SD}=70 \mathrm{~ms}$ ) conditions (ps $<0.001)$, which in turn did not differ from each other.

\section{Intentional binding (time-judgement errors)}

Finally, the ANOVA on the time-judgment errors revealed a significant effect of Congruency $\left(F_{(2,54)}=3.18 ; p<0.05 ; \eta 2 p=0.11\right)$. Incongruent condition (Mean $=-62 \mathrm{~ms} ; \mathrm{SD}=74 \mathrm{~ms}$ ) led to significantly greater interval estimates, and consequently to reduced intentional binding, as compared to congruent condition (Mean $=-72 \mathrm{~ms} ; \mathrm{SD}=81 \mathrm{~ms})(\mathrm{p}=0.04)$, while a marginally significant difference was observed between congruent and baseline conditions (Mean $=-61 \mathrm{~ms}$; $\mathrm{SD}=80 \mathrm{~ms})(\mathrm{p}=0.06)$. 


\subsection{Electrophysiological Results and ERP-Behavior Correlation}

\subsubsection{Target ERPs}

The mass univariate analysis of the main effect of Condition revealed a number of significant differences (critical $\mathrm{F}_{(2,54)}=3.57$ ). Based on their distinct spatio-temporal characteristics these ERP differences were grouped into four main clusters, as detailed below (see Fig. 2).

<Fig. 2 here> Figure 2. Electrophysiological results, Target epoch. Raster diagram showing significant differences across ERPs elicited by the three experimental conditions according to the TFCE permutation test (see text). Channels are represented on the $\mathrm{y}$ axis and time points on the $\mathrm{x}$ axis (in ms relative to the target onset). Rectangles in warm colors indicate channels/time points showing significant condition-dependent ERP differences corrected for multiple comparisons. The color bar on the right indicates $\mathrm{F}$ values. Green rectangles indicate electrodes/time points showing no significant differences.

The first significant ERP effect was observed between 130 and $160 \mathrm{~ms}$ and concerned an early positive ERP component distributed over occipito-parietal electrodes. We observe increased (more positive) amplitudes for both Congruent and Incongruent relative to the Baseline condition (Fig. 3A, B). Based on its spatio-temporal pattern, this ERP effect can be described as a stronger visual P100 component for non-Baseline conditions. Concurrently with this we observe an increased (more negative) ERP amplitude for both Congruent and Incongruent relative to the Baseline condition over frontal electrodes. (Fig. 3A, C).

<Fig 3 here> Figure 3. Electrophysiological results, Target epoch, P100 effect. The topoplot in (A) shows the scalp distribution of the F values for the main effect of congruency on ERP amplitudes corresponding to the time point when the P100 effect revealed by the TFCE permutation test was maximal $(148 \mathrm{~ms}$, also see the dotted vertical line in plots depicted in panels B and C). Channels belonging to spatio-temporal cluster showing significant effects are depicted as black squares. (B, C) The plots show the ERPs for the three experimental conditions averaged across channels showing maximal effects: POz, PO3, and PO4 for (B) and AFz, AF3, and AF4 for (C).

The second significant ERP effect was observed between 180 and $210 \mathrm{~ms}$ and concerned a negative ERP component distributed over left and right occipito-temporal electrodes. We observe increased (more negative) amplitudes for both Congruent and Incongruent relative to the Baseline condition (Fig 4A, B). Based on its spatio-temporal pattern, this ERP effect can be described as a stronger N200 component for non-Baseline conditions. Concurrently with this we observe an increased (more positive) ERP amplitude for both Congruent and Incongruent relative to the Baseline condition over fronto-central electrodes (Fig 4A, C).

<Fig 4 here> Figure 4. Electrophysiological results, Target epoch, N200 effect. The topoplot in (A) shows the scalp distribution of the $\mathrm{F}$ values for the main effect of congruency on ERP amplitudes corresponding to the time point when the N200 effect revealed by the TFCE permutation test was maximal (201 ms, see the dotted vertical line in plots depicted in panels B and C). Channels belonging to spatio-temporal cluster showing significant effects are depicted as black squares. $(B, C)$ The plots show the ERPs for the three experimental conditions averaged across channels showing maximal effects: P10, P8, PO9 and P7 for (B) and FCz, FC1, and FC2 for (C). 
Interestingly, the third significant ERP effect was observed between 250 and $350 \mathrm{~ms}$ peaking around $300 \mathrm{~ms}$ over centro-parietal electrodes. We observe increased (more positive) amplitudes for both Congruent and Incongruent relative to the Baseline condition (Fig. 5A). The spatiotemporal pattern of this ERP suggest that belong to a typical P300 component. In addition, we observe a cluster of activities with increased amplitude for the Congruent relative to the Incongruent condition between 300 and $350 \mathrm{~ms}$ (Fig. 5B). The amplitude of this P300 effect was the highest for the Congruent condition, it was followed by the Incongruent condition, and finally by the Baseline condition. Concurrently with this we observe an increased (more negative) ERP amplitude for both Congruent and Incongruent relative to the Baseline condition left and right temporal channels (Fig. 5A, C).

$<$ Fig 5 here> Figure 5. Electrophysiological results, Target epoch, P300 effect. The topoplot in (A) shows the scalp distribution of the $\mathrm{F}$ values for the main effect of congruency on ERP amplitudes corresponding to the time point when the P300 effect revealed by the TFCE permutation test was maximal $(306 \mathrm{~ms}$, , see the dotted vertical line in plots depicted in panels B and C). Channels belonging to spatio-temporal cluster showing significant effects are depicted as black squares. $(\mathrm{B}, \mathrm{C})$ The plots show the ERPs for the three experimental conditions averaged across channels showing maximal effects: $\mathrm{CPz}, \mathrm{CP} 1$, and $\mathrm{CP} 2$ for $(\mathrm{B})$ and $\mathrm{P} 10, \mathrm{P} 8, \mathrm{PO} 9$ and $\mathrm{P} 7$ for $(\mathrm{C})$. The dotted line indicate the time point when the significant difference between Congruent and Incongruent trials was maximal.

A fourth significant ERP effect was observed in a later time widow starting around $400 \mathrm{~ms}$ and likely represents the persistence of the P300 effect. As before, we observe increased (more positive) amplitudes for both the Congruent and Incongruent relative to the Baseline condition (Fig 6A, B). Concurrently with this we observed increased (more negative) ERP amplitudes for both the Congruent and Incongruent relative to the Baseline condition over temporal electrodes. The mass-univariate correlation analysis revealed that differences between the Congruent and the Baseline condition in this late P300 were positively correlated with the Behavioral differences (intentional binding measures) between the Congruent and the Baseline condition between 370 and $430 \mathrm{~ms}$. No significant correlations were observed for the Incongruent-Baseline differences (Fig. 6C, D). Importantly, a Steiger's Z test for the comparison between correlations revealed that the peak brain-behavior correlation for the difference Congruent-Baseline was significantly different from the difference Incongruent-Baseline $(\mathrm{Z}=2.17, \mathrm{p}=.030)$, showing that the brainbehavior correlation was specific for the Congruent condition rather than a non-Baseline effect.

<Fig 6 here> Figure 6. Electrophysiological results, Target epoch, late P300 effect. The topoplot in (A) shows the scalp distribution of the F values for the main effect of congruency on ERP amplitudes corresponding to the time point when the late central effect revealed by the TFCE permutation test was maximal ( $414 \mathrm{~ms}$, also see the dotted vertical line in plots depicted in panels B). Channels belonging to spatio-temporal cluster showing significant 
effects are depicted as black squares. The plot in (B) shows the ERPs for the three experimental conditions averaged across $\mathrm{CPz}, \mathrm{CP} 1$, and $\mathrm{CP} 2$ channels. The scatterplot in (C) shows the significant brain-behavior correlation between the Congruent vs. Baseline behavioral effect ( $y$ axis) and the corresponding ERP effect extracted from the centroparietal channel (CP2) and time point ( $420 \mathrm{~ms}$ ) showing the maximal effect ( $x$ axis). The scatterplot in (D) shows the non-significant brain-behavior correlation between the Incongruent vs. Baseline behavioral effect and the corresponding ERP effect extracted from the same channel and time point used in (C).

\subsubsection{Pre-response ERPs}

The mass univariate analysis of the main effect of Condition revealed a number of significant differences (critical $\mathrm{F}_{(2,54)}=3.64$ ) near the time of the response. Based on their distinct spatiotemporal characteristics these ERP differences were grouped into three main clusters, as detailed below (see Fig. 7).

$<$ Fig 7 here> Figure 7. Electrophysiological results, Pre-response epoch. Raster diagram showing significant differences across ERPs elicited by the three experimental conditions according to the TFCE permutation test (see text). Channels are represented on the $y$ axis and time points on the $x$ axis (in ms relative to the response). Rectangles in warm colors indicate channels/time points showing significant condition-dependent ERP differences corrected for multiple comparisons. The colorbar on the right indicates F values. Green rectangles indicate electrodes/time points showing no significant differences.

A significant ERP effect was observed around $350 \mathrm{~ms}$ before the response and corresponded to a pre-response positivity distributed over centro-parietal electrodes. We observe an early and larger response for Incongruent relative to both the Congruent and Baseline conditions, an effect that was sustained until the response time (Fig. 8A, B). The mass-univariate correlation analysis revealed that ERP differences between the Incongruent and the Congruent condition were negatively correlated with the Behavioral differences (intentional binding measures) between the Incongruent and the Congruent condition around $270 \mathrm{~ms}$ before the response and between 150 and $30 \mathrm{~ms}$ before the response, while the brain behavior correlations involving the IncongruentBaseline and Congruent-Baseline differences were not significant (Fig. 8C, D). Opposite correlations were found for the Incongruent-dependent negativity over left anterior-frontal electrodes in similar time windows.

<Fig 8 here> Figure 8. Electrophysiological results, Pre-response epoch, pre-response positivity effect. The topoplot in (A) shows the scalp distribution of the F values for the main effect of congruency on ERP amplitudes corresponding to the time point when the early centro-parietal positivity effect revealed by the TFCE permutation test was maximal (-336 ms, see the dotted vertical line in plots depicted in panels B). Channels belonging to spatiotemporal cluster showing significant effects are depicted as black squares. The plot in (B) shows the ERPs for the three experimental conditions averaged across $\mathrm{CPz}, \mathrm{CP} 1$, and $\mathrm{CP} 2$ channels. Note that the effect lasted until around the time of the response. The scatterplot in (C) shows the significant brain-behavior correlation between the Incongruent vs. Congruent behavioral effect ( $y$ axis) and the corresponding ERP effect ( $x$ axis) extracted from the 
centro-parietal channel $(\mathrm{CPz})$ and time point $(-140 \mathrm{~ms})$ showing the maximal effect. The scatterplot in (D) shows the non-significant brain-behavior correlation between both the Incongruent vs. Baseline and the Congruent vs. Baseline behavioral effects and the corresponding ERP effects extracted from the same channel and time point used in $(\mathrm{C})$.

At the same time, $280 \mathrm{~ms}$ before the response the ERP effects spread over frontal electrodes and, gradually, all over the scalp, with a complex spatio-temporal pattern. Specifically, more anterior electrodes showed a sustained negativity while parietal electrodes showed a sustained positivity between 200 and $100 \mathrm{~ms}$ before the response. These responses were significantly greater for the Incongruent as compared to both the Baseline and Congruent conditions, and also greater for the Baseline as compared to the Congruent condition (Fig. 9 A-C).

<Fig 9 here> Figure 9. Electrophysiological results, Pre-response epoch, anterior negativity. The topoplot in (A) shows the scalp distribution of the F values for the the main effect of congruency on ERP amplitudes corresponding to the time point when the anterior negativity effect revealed by the TFCE permutation test was maximal (-147 ms). Channels belonging to spatio-temporal cluster showing significant effects are depicted as black squares. The plots in $(\mathrm{B}, \mathrm{C})$ show the ERPs for the three experimental conditions averaged across channels showing maximal effects: P1 and P3 for (B) and F3 and AF3 for (C). The dotted line indicate the time point when the significant difference across condition was maximal.

\subsubsection{Post-response ERPs}

The mass univariate analysis of the main effect of Condition revealed a number of significant differences (critical $\mathrm{F}_{(2,54)}=3.64$ ) that were continuous in time after the response. Based on their distinct spatio-temporal characteristics ERP differences were grouped into three main clusters, as detailed below (see Fig. 10).

<Fig 10 here> Figure 10. Electrophysiological results, Post-response epoch. Raster diagram showing significant differences across ERPs elicited by the three experimental conditions according to the TFCE permutation test (see text). Channels are represented on the $\mathrm{y}$ axis and time points on the $\mathrm{x}$ axis (in ms relative to the response). Rectangles in warm colors indicate channels/time points showing significant condition-dependent ERP differences corrected for multiple comparisons. The colorbar on the right indicates F values. Green rectangles indicate electrodes/time points showing no significant differences.

A significant ERP effect was observed between -30 and $30 \mathrm{~ms}$ around the response time and concerned a positive ERP distributed over centro-parietal electrodes. These responses were the largest (more positive) for the Congruent condition, followed by the baseline condition and finally 
by the Incongruent condition. (Fig. 11A, B). Concurrent ERP responses over frontal electrodes mirrored this pattern of results (Fig. 11A, C).

<Fig 11 here> Figure 11. Electrophysiological results, Post-response epoch, peri-response effect. The topoplot in (A) shows the scalp distribution of the $\mathrm{F}$ values for the significant differences across ERPs elicited by the three experimental conditions corresponding to the time of the response $(0 \mathrm{~ms}$, also see the dotted vertical line in plots depicted in panels B). Channels belonging to spatio-temporal cluster showing significant effects are depicted as black squares. The plots in (B, C) show the ERPs for the three experimental conditions averaged across channels showing maximal effects: CPz, CP1 and CP2 for (B) and F7, FT7 and FC5 for (C).

A second significant ERP effect was observed between 40 and $70 \mathrm{~ms}$ and was characterized by a widespread distribution over the scalp with a single spatial cluster of significant electrodes. We observe increased negative ERP responses for Incongruent as compared with both Congruent and Baseline conditions over central sites (Fig. 12A, B). In addition, the mass-univariate correlation analysis revealed that all the three ERP amplitude differences (Incongruent-Congruent, Incongruent- Baseline, and Congruent-Baseline) over $\mathrm{CPz}$ and $\mathrm{CP} 1$ were positively correlated with the corresponding behavioral effects (intentional binding measures) (Fig. 12C-E). At the same time, ERP differences were observed over anterior channels with more negative ERP amplitudes for both Congruent and Incongruent as compared to the Baseline condition. The massunivariate correlation analysis revealed that all the three ERP effects differences (IncongruentCongruent, Incongruent-Baseline, and Congruent-Baseline) over left fronto-temporal electrodes were negatively related to the corresponding behavioral effects (intentional binding measures). Lastly, we observed increased positive posterior ERP responses for both Congruent and Incongruent as compared to the Baseline condition.

<Fig 12 here> Figure 12. Electrophysiological results, Post-response epoch, early centro-parietal effect. The topoplot in (A) shows the scalp distribution of the $\mathrm{F}$ values for the significant differences across ERPs elicited by the three experimental conditions corresponding to the time point when the early centro-parietal effect revealed by the TFCE permutation test was maximal $(63 \mathrm{~ms})$. Channels belonging to spatio-temporal cluster showing significant effects are depicted as black squares. The plot in (B) shows the ERPs for the three experimental conditions averaged across $\mathrm{CPz}$ and $\mathrm{CP} 1$ channels. The scatterplot in $(\mathrm{C})$ shows the significant brain-behavior correlation between the Incongruent vs. Congruent behavioral effect ( $y$ axis) and the corresponding ERP effect ( $x$ axis) extracted from the centro-parietal channels $(\mathrm{CPz})$ and time point $(50 \mathrm{~ms})$ showing the maximal effect (see the vertical dotted line in b). The scatterplots in (D) and (E) show, respectively, the significant brain-behavior correlation between the Incongruent vs. Baseline and the Congruent vs. Baseline behavioral effects and the corresponding ERP effects extracted from the same channel and time point used in (C). 
The last significant ERP effect was observed in a time widow starting from $160 \mathrm{~ms}$ and lasting until the end of the epoch. Generally, this effect was characterized by similar scalp topography and ERP amplitudes as compared with the effects described in the previous paragraph and could be considered as their continuation.

\section{Discussion}

In the present study we aimed at investigating electrophysiological correlates of the fluency of action and how they were related to intentional binding. It is important to note that we refer to the fluency of action selection and not to the fluency between actions and outcomes. Therefore, we primarily focused on response preparation and execution processes prior the sensory consequences of the actions. We recorded EEG signals while participants performed an imitation-inhibition task in combination with a time estimation task. Participants performed an action (key release) and in parallel they observed an actor performing their same movement (congruent condition), or an opposite movement (incongruent condition) or no movement (baseline condition). Their action produced an effect (auditory effect), and we asked them to judge the intervals between their action and the ensuing effect. In line with our previous behavioral findings (Vastano et al., 2017) we replicated a larger intentional binding effect for Congruent relative to Incongruent trials. Consistent with a fluency account of SoA (Valerian Chambon \& Haggard, 2012; Valérian Chambon et al., 2014; Wenke, Fleming, \& Haggard, 2010) our results demonstrate the relevance of a correspondence between intention and action and, thus, of a coherent integration of action representations for the experience of agency. In order to investigate the impact of conscious processing of stimulus-congruency and response-fluency on the intentional binding we focused on EEG components associated with target and response processing, respectively.

\subsection{Target processing and SoA}

Components that showed a difference between the two movements conditions and baseline:

The stimulus display elicited two early brain responses (i.e. a P100 and a N200) most likely related to attention and processing of biological motion. The first component was observed between 130$160 \mathrm{~ms}$ on bilateral occipito-parietal electrodes. The amplitude of this visual P100 response was larger for both congruent and incongruent compared with Baseline trials. This effect seems to reflect a stronger visual processing for trials where an imperative stimulus (in both Congruent and Incongruent trials) was presented as compared with trials where no finger movement was presented (Baseline condition). This interpretation is line with previous ERP studies that show 
enhanced P100 response for stimuli bearing a distinctive feature (such as a specific color) that

A second negative ERP response was observed between 180 and $210 \mathrm{~ms}$ over bilateral occipitotemporal sites. The amplitude of this response was higher for both congruent and incongruent compared with Baseline trials. Previous studies have shown that the observation of human forms and biological motion evokes a negative response peaking around 170-200 ms post-stimulus onset maximal over posterior occipito-temporal electrodes (Hirai, Fukushima, \& Hiraki, 2003; Hirai, Senju, Fukushima, \& Hiraki, 2005; Krakowski et al., 2011; White, Fawcett, \& Newman, 2014). In line with this evidence, in our study the observation of finger movement (in both Congruent and Incongruent trials) induced a larger N200 response irrespectively if they were congruent or incongruent with the imperative cue, thus suggesting that this response is selective for biological motion processing.

Finally and more interestingly, we observe later components related to high-level cognitive processes.

The results showed first a centro-parietal positive ERP response that peaked around $300 \mathrm{~ms}$. The amplitude of this P300 response was larger for both congruent and incongruent compared with baseline trials. This $\mathrm{P} 300$ effect seems to represent a surprise effect or mismatch detection process reminiscent of evoked P300-like responses for deviant stimuli (Polich, 2007). The surprise effect on the P300 amplitude has been located at the anterior cingulate cortex and is interpreted as a disruption of attentional resources by unexpected stimuli appearing among standard stimuli (Huster, Westerhausen, Pantev, \& Konrad, 2010; Polich, 2007). The central distribution and short latency of our P300 fit the description of this effect. Our Baseline stimuli are unexpected events because, contrary to the other conditions (the Congruent and the Incongruent) no motion was displayed. In addition, from the total of trials, the Baseline trials were less probable than the trials showing finger movements (Congruent and Incongruent trials summed together).

Component revealing a difference between congruent and incongruent trials:

Importantly, a later but similar ERP response was found in this spatial configuration between 300$350 \mathrm{~ms}$. This response was also larger for both Congruent and Incongruent compared with Baseline trials, but crucially we observe a larger response for the Congruent compared with Incongruent and Baseline trials. Previous studies in social cognitive neuroscience have suggested a link between P300 and self-other distinction processes (Deschrijver, Wiersema, \& Brass, 2015; Deschrijver et al., 2017; Holeckova, Fischer, Giard, Delpuech, \& Morlet, 2006; Longo, Musil, \& Haggard, 2012). A previous study on the imitation-inhibition task showed a larger P300 amplitude 
for the Congruent relative to Incongruent and Baseline trials and was interpreted in terms of selfmechanism was required to distinguish the intended from the externally triggered motor plans, with the congruency effects reflecting conflict between two motor plans. Our findings of enhanced responses for the Congruent relative to the Incongruent and Baseline conditions for this late P300 are in agreement with these previous findings. However, we note some distinctions regarding the current and this previous study. Our data-driven approach aimed at investigating differences in the entire spatio-temporal domain instead of focusing in selected a priori time windows. Despite the different approaches, our results converge with the results of Deschrijver et al. (2017), with larger responses for Congruent trials. The P300 described there, would correspond to our component that here we labeled as a late P300 (300-350 ms), since statistical differences in Deschrijver et al. (2017) were found in a time-window ranging from 310 to $430 \mathrm{~ms}$. Although these results can be interpreted in terms of self-other distinction processes we might also consider other important interpretations.

Previous studies have suggested that the P300 component might reflect a measure of mental workload. These studies have shown decreased amplitude and latency of the P300 for tasks with high difficulty (for a review see (Kok, 2001), such as in paradigms involving stimulus-response incompatibility (Ragot \& Fiori, 1994; Sebanz et al., 2006), complex conceptual operations (García-Larrea \& Cézanne-Bert, 1998; Ullspererg, Metz, \& Gille, 1988), and response complexity (Ragot \& Fiori, 1994; Ragot \& Renault, 1981). These studies suggest that smaller P300 amplitudes for difficult conditions might represent a reduction of attentional resources due to high cognitive load (Kok, 2001; Polich, 2007). More importantly, studies on stimulus-response compatibility, like our paradigm, showed a larger P300 amplitude on trials where the target was congruent relative to incongruent with the performed action (Deschrijver et al., 2017; Ragot \& Fiori, 1994; Sebanz et al., 2006). Our results converge with this evidence and is supported by the fact that congruent trials were responded faster and were less prone to errors (that is, have a low cognitive load) as compared with baseline and incongruent trials. Interestingly, we found that ERP differences for the late P300 (420 ms) between the Congruent and the Baseline condition were positively correlated with the same Behavioral differences (Congruent - Baseline intentional binding measures). Thus, participants with larger differences (Congruent - Baseline) in the late P300 have also larger differences (Congruent-Baseline) in judgment errors, and thus stronger differences in implicit SoA. This is a specific effect for the Congruent condition since the other brain-behavior correlation involving the Incongruent condition was non-significant and significantly smaller. 
These findings suggest that a perceived stimulus that reduces the cognitive load by facilitating the performance impacts positively the SoA through a P300 effect. This interpretation is reinforced by the positive correlation between the difference in P300 amplitude and the difference in intentional binding (congruent dependent effect). In addition, we suggest that this late P300 might represent an electrophysiological marker associated with SoA: for tasks where observed targets do not interfere with the to-be performed actions, and thus have reduced cognitive load, we can expect larger late P300 amplitudes reflecting stronger intentional binding effects. To our knowledge, this is the first study showing a link between a supraliminal target processing and the intentional binding. Our results put forward the pivotal role of conscious elaboration of the external environment on response preparation impacting the subsequent, more cognitive, SoA.

An alternative explanation that also fits our results, is that a high late P300 amplitude in the Congruent condition might result from higher motivational significance of the stimulus and paid attention. In order to perform the time estimation task we speculate that participants might have focused on the contingencies between the target and action outcome. In this scenario, the Congruent trials represent relevant and positive stimuli that can involve higher attentional resources. Thus, congruent trials represent a positive significance and capture more attention for the execution of the secondary task. In line with these ideas, it has been shown that the P300 reflects the phasic activity of the neuromodulatory locus coeruleus (LC)-norepinephrine system (NE) (Nieuwenhuis, Aston-Jones, \& Cohen, 2005). LC phasic response is driven by salient stimuli and task-relevant decisional processes that in turn facilitate the performance, as also shown for the P300 response. Although here we cannot provide a direct link between LC phasic response and P300, we speculate that congruent trials enhanced the LC phasic activity producing higher P300 amplitude and a better performance as reflected by faster RTs and higher accuracy for these trials.

Overall, the reduced cognitive load and the high motivational significance of a congruent supraliminal perceived stimulus would induce participants to perceive positively the target-actionoutcome link signaling a larger P300 effect associated with a stronger SoA.

\subsection{Pre-response effects and SoA}

Responses to the stimulus displays were associated with positive pre-response ERP activities that covered centro-parietal electrodes. The amplitude of these ERP responses were larger for Incongruent compared with Congruent and Baseline trials and was sustained from $350 \mathrm{~ms}$ before the response until the moment of the response. A centro-parietal positive ERP previous to a movement was originally described by Deecke et al.1969 as a motor potential occurring before 
movement onset and reflecting processes related to voluntary self-triggered movement initiation (Deecke, Scheid, \& Kornhuber, 1969). Our study show some convergence with this notion in the sense that our task required voluntary actions. However, these actions were triggered by an external stimulus (a target number) and were associated with conflictive (incongruent trials) and non-conflictive (congruent and baseline trials) situations. A more recent study (Lucci, Berchicci, Spinelli, \& Di Russo, 2014) compared compatible and incompatible movements: drawing simultaneously with both hands a circle or a vertical line and drawing simultaneously a circle with the left hand and a vertical line with the right hand or vice versa, respectively. The authors showed a larger central positivity for incompatible relative to compatible movements two seconds before the movement until movement onset. A source analysis identified the lateral premotor cortex (PMAd; BA 6) of both hemispheres as a cortical source of the central positivity for incompatible movements. The authors concluded that incompatible and anti-phase movements that generated the pre-movement central positivity activated a well-known "interference network" which engages different motor areas, such as the premotor cortex, the bilateral supplementary motor area (SMA), the cingulate motor area (CMA), and the bilateral parietal cortex (Ehrsson, KuhtzBuschbeck, Forssberg, Gerloff, \& Hummel, 2002; Sadato, Yonekura, Waki, Yamada, \& Ishii, 1997). This evidence supports the notion that the larger positive pre-response ERP activities (at $\mathrm{Cz}$ ) for the Incongruent condition relative to the Congruent and Baseline ones in our paradigm are associated with the complexity of motor preparation and interference processes for Incongruent trials. Interestingly, we also observe that pre-response ERP differences (around $270 \mathrm{~ms}$ before the response) between the Incongruent and the Congruent condition were negatively correlated with the Behavioral differences (intentional binding measures) between the Incongruent and the Congruent condition. Thus, participants with larger differences (Incongruent-Congruent) in this pre-response central-positivity have smaller differences (Incongruent-Congruent) in timejudgment errors. We believe that this result is driven by the incongruent condition and interference processes. In other words, when the congruency-effect is reduced the motor interference preresponse increases generating higher pre-response positivity. We conclude that pre-movement interference processes typical of incongruent trials has a negative impact on the intentional binding effect. The electrophysiological marker of this effect is represented by higher preresponse central positivity.

We also found a frontal negativity with an increased ERP response for Incongruent relative to Congruent trials around $280 \mathrm{~ms}$ before the response at frontal electrodes (Fig. 9C). This result is reminiscent of the effects found for the readiness potential (RP) in a previous study of the imitation-inhibition paradigm (Deschrijver et al., 2017). We think that this ERP effect reflects 
motor preparation processes: in the Congruent condition the facilitation of the participant's selection is interfered.

\subsection{Post-response effects and SoA}

Responses to the stimulus displays were associated with negative post-response ERP activities that covered centro-parietal electrodes during the time of the response until $70 \mathrm{~ms}$ later. The amplitude of these ERP responses were larger for Incongruent compared with Congruent and Baseline trials (early centro-parietal effect - Fig.12). Although a bit more central than usual the observed topography and timing suggest the presence of a correct-related negativity-like (CRNlike) potential. The CRN is a fronto-central ERP response that appear within a short time window of $100 \mathrm{~ms}$ after a correct response (Coles, Scheffers, \& Holroyd, 2001; Vidal, Hasbroucq, Grapperon, \& Bonnet, 2000). The CRN is said to reflect response evaluation processes, in particular for uncertainty about correct response and post-response conflict monitoring (Grützmann et al., 2014; Kwapil et al., 2008; Pailing \& Segalowitz, 2004). In our paradigm the Incongruent condition might have induced uncertainty in the response due to conflicting information from the observed stimulus. This uncertainty might have elicited an increased negative CRN-like response for Incongruent compared with Baseline and Congruent conditions. While our primary focus was on motor preparation and action execution mechanisms, rather than on processing of action effects, we can also consider an alternative explanation related to the expectation of the outcomes. Participants' response was always followed by an auditory outcome to which participants are asked to respond to complete the secondary time estimation task, and therefore highly expected. The auditory outcome was delivered after participants' response (300 $\mathrm{ms}$ later at the shortest interval). In this context, one possibility is that post-response ERP activities (early centro-parietal effect) can also reflect stimulus anticipation processes. Our analysis showed that slow negative ERPs unfolded in time at central electrodes with more negative responses for the Incongruent condition and continue to be negative for all of the three conditions until the end of the epoch. Slow negative potentials (at central electrodes) preceding stimulus onset have been shown to be related to anticipation processes, such as anticipation of probes, and affective and informative stimuli (van Boxtel \& Böcker, 2004). The contingent negative variation (CNV) is a negative potential recorded on the vertex that appears before an imperative expected stimulus at which participants need to react (Walter, Coorper, Aldrige, McCallium, \& Winter, 1964). The CNV has been also linked to arousal, motivation, and information processing (Tecce, 1972). Since in our paradigm responses were always followed by the auditory outcome, these stimuli could be 
expected by the participants in order to perform the secondary time-estimation task, with more anticipation for Incongruent trials, given their conflictual characteristic.

Finally, differences in post-response ERPs activities (early centro-parietal effect) were correlated with intentional binding effects. We found positive correlations between all the three ERP differences (Incongruent-Congruent, Incongruent-Baseline, and Congruent-Baseline) and the corresponding behavioral effects (intentional binding measures) (Fig. 12). Thus, participants with larger differences in this negative ERP have also larger differences in judgment errors, and thus stronger differences in implicit SoA. Since these correlations were unspecific to conditions, these results suggest that the central post-response ERP effects might indeed reflect expectation processes common to all conditions.

\section{Conclusions and future directions}

In this study we were interested in understanding whether intentional binding is primarily driven by fluency induced by stimulus processing and response preparation or by processes following response execution. We provide evidence that both pre and post-response mechanisms are important in the generation of the intentional binding. However, stimulus processing and preresponse mechanisms are the only ones showing specific brain-behavioral correlations.

To summarize, congruent with our predictions, we found that the fluency in action preparation has an influence on the intentional binding at the stimulus processing level, as shown by the late P300 modulated by the congruency-effect. The implicit SoA is therefore modulated by processes occurring also during the early stimulus processing stages, and it is boosted in situations where no interference occurs reducing the cognitive load. This conclusion is also based on the correlational result between the late P300 and intentional binding specific for congruent conditions.

In concordance with previous fluency account of agency (Valerian Chambon \& Haggard, 2012; Sidarus, Vuorre, Metcalfe, \& Haggard, 2017) we found an effect of congruency also at the stage of action selection. Pre-response ERP activities (centro-parietal positivity) were enhanced for Incongruent trials, and possibly signal motor interference processes. A negative correlation confirmed this interpretation by showing higher centro-parietal positivity for reduced congruencyeffects in the intentional binding (incongruent-dependent effect).

Finally, the post-response ERPs also showed a larger ERP response (a central negativity) for Incongruent trials, an affect that seems to be associated with response uncertainty, however we fail to find a significant correlation selective for an incongruent-dependent effect. But we found that this post-response central negativity was positively correlated with the correspondent 
intentional binding effect in all the three conditions, presumably providing evidence for actionoutcomes anticipation processes.

Altogether, our study sheds new light regarding the neural correlates of the implicit SoA modulated by the action-congruency effects (using a supraliminal action-prime), as related to fluency of action.

A final observation that needs to be pointed out is that we used intentional binding that represents only an implicit measure of agency and the simple causal relationship between actions and effects has an influence on this mechanism (Buehner, 2012; Buehner \& Humphreys, 2009). However it has been shown that causality between events is not enough to generate the intentional binding effect, a strong effect requires that events in the external environment have to be recognized as consequences of one's action (Buehner 2015; Cravo, Claessens, \& Baldo, 2009; Engbert, Wohlschläger \& Haggard, 2008). Although we do not test explicit judgments of agency, previous studies on explicit measures and fluency of actions show similar results to ours, that is the explicit sense of agency is reinforced when there is not discrepancy at the action selection level (Chambon \& Haggard, 2012; Chambon, Sidarus, \& Haggard, 2014; Sidarus \& Haggard, 2016), this leads to the assumption that the fluency of action selection similarly affects explicit judgments of agency and the intentional binding effect. Our results, therefore, fit within agency mechanisms, adds to the existing literature on agency and opens new avenues for understanding how human behavior relates to the feelings of agency under conflict situations generated during preparatory and postaction stages.

Nevertheless, future studies may address new questions on the topic of agency during incongruent situations and dysfluent mechanisms. For example, one could argue that when acting incongruently with an external stimulus there is more voluntariness that reinforces the subjective experience of being in control and as a consequence feeling more agency. This is because participants may experience to act in opposition to a stimulus or event to achieve an effect. Similarly, studies on effort showed that actions involving physical effort to cause an effect enhanced intentional binding, increasing the perception of self as intentional agents compared with actions without effort (Demanet, Muhle-Karbe, Lynn, Blotenberg \& Brass, 2013; Preston \& Wegner, 2007; 2009). Differently, our study together with other studies on fluency of action selection (Chambon \& Haggard, 2012; Chambon, Sidarus, \& Haggard, 2014; Sidarus \& Haggard, 2016) show less agency and poorer performance for conditions where there is a higher cognitive load during action planning (incongruent conditions). We believe that on the action selection level participants may not experience to act consciously 
in opposition to an external event to generate outcomes, especially if these latter are equally predicted across conditions, like in our task. Our participants may experience mainly interference and uncertainty during action planning that reduces the subjective experience of being in control. Our aim was limited to understand neural correlates of intentional binding during the fluency of action selection. However, the use of different approaches to manipulate action-congruency and conflict based on different levels of action awareness may lead to different results. Therefore new studies are needed to advance knowledge on brain mechanisms of agency when external events for which we are aware impact the facilitation of obtaining expected effects and as a consequence the belief of being an agent.

\section{Author contributions}

MB, RV and JLUF conceived and designed the study. JLUF and RV collected the data. AE and $\mathrm{RV}$ analyzed the data and wrote the first draft of the manuscript. MB supervised the research and gave important contribution for drafting the final version of the manuscript. All authors contributed to revise the manuscript and approved the final version.

\section{Conflict of interest}

The authors declare no conflict of interest.

\section{Acknowledgements}

This research did not receive any specific grant from funding agencies in the public, commercial, or not-for-profit sectors

\section{References}

Bednark, J. G., \& Franz, E. A. (2014). Agency attribution: Event-related potentials and outcome monitoring. Experimental Brain Research, 232, 1117-1126. https://doi.org/10.1007/s00221-014-3821-4

Brass, M., Bekkering, H., \& Prinz, W. (2001). Movement observation affects movement execution in a simple response task. Acta Psychologica, 106(1-2), 3-22. https://doi.org/10.1016/S0001-6918(00)00024-X

Brass, M., Bekkering, H., Wohlschläger, A., \& Prinz, W. (2000). Compatibility between observed and executed finger movements: comparing symbolic, spatial, and imitative cues. Brain and Cognition, 44(2), 124-143. https://doi.org/10.1006/brcg.2000.1225 
Brass, M., Derrfuss, J., \& von Cramon, D. Y. (2005). The inhibition of imitative and overlearned

Buehner, M.J. \& Humphreys G.R.(2009).Causal binding of actions to their effects: Research article. Psychological Science, 20, 1221-1228, 10.1111/j.1467-9280.2009.02435.x

Buehner M.J. (2012). Understanding the past, predicting the future: Causation, not intentional action, is the root of temporal binding. Psychological Science, 23(12),1490-1497 https://doi.org/0.1177/0956797612444612

Buehner, M. J. (2015). Awareness of voluntary and involuntary causal actions and their outcomes. Psychology of Consciousness: Theory, Research, and Practice, 2(3), 237-252. https://doi.org/10.1037/cns0000068

Caspar, E. A., Cleeremans, A., \& Haggard, P. (2015). The relationship between human agency and embodiment. Consciousness and Cognition, 33, 226-236. https://doi.org/10.1016/j.concog.2015.01.007

Chambon, V., \& Haggard, P. (2012). Sense of control depends on fluency of action selection, not motor performance. Cognition, 125(3), 441-451. https://doi.org/10.1016/j.cognition.2012.07.011

Chambon, V., Sidarus, N., \& Haggard, P. (2014). From action intentions to action effects: how does the sense of agency come about? Frontiers in Human Neuroscience, 8(May), 320. https://doi.org/10.3389/fnhum.2014.00320

Cohen, J. (1988). Statistical power analysis for the behavioral sciences. Statistical Power Analysis for the Behavioral Sciences. https://doi.org/10.1234/12345678

Coles, M. G. H., Scheffers, M. K., \& Holroyd, C. B. (2001). Why is there an ERN/Ne on correct trials? Response representations, stimulus-related components, and the theory of errorprocessing. Biological Psychology, 56(3), 173-189. https://doi.org/10.1016/S03010511(01)00076-X

Cravo, A. M., Claessens, P. M. E., \& Baldo, M. V. C. (2009). Voluntary action and causality in temporal binding. Experimental Brain Research, 199, 95-99. https://doi.org/10.1007/s00221-009-1969-0

David, N., Newen, A., \& Vogeley, K. (2008). The "sense of agency" and its underlying 
cognitive and neural mechanisms. Consciousness and Cognition, 17(2), 523-534. https://doi.org/10.1016/j.concog.2008.03.004

Deecke, L., Scheid, P., \& Kornhuber, H. H. (1969). Distribution of readiness potential, premotion positivity, and motor potential of the human cerebral cortex preceding voluntary finger movements. Experimental Brain Research, 7(2), 158-168. https://doi.org/10.1007/BF00235441

Delorme, A., Fernsler, T., Serby, H., \& Makeig, S. (2006). EEGLAB Tutorial Table of Contents. San Diego California University of San Diego, 1-18. https://doi.org/10.1093/carcin/bgs090

Delorme, A., \& Makeig, S. (2004). EEGLAB: An open source toolbox for analysis of singletrial EEG dynamics including independent component analysis. Journal of Neuroscience Methods, 134(1), 9-21. https://doi.org/10.1016/j.jneumeth.2003.10.009

Demanet, J., Muhle-Karbe, P.S., Lynn, M.T., Blotenberg, I., \& Brass M. (2013). Power to the will: how exerting physical effort boosts the sense of agency. Cognition, 129(3),574-578. https://doi.org/10.1016/j.cognition.2013.08.020

Deschrijver, E., Wiersema, J. R., \& Brass, M. (2015). The interaction between felt touch and tactile consequences of observed actions: An action-based somatosensory congruency paradigm. Social Cognitive and Affective Neuroscience, 21. Retrieved from http://scan.oxfordjournals.org/

Deschrijver, E., Wiersema, J. R., \& Brass, M. (2017). The influence of action observation on action execution: Dissociating the contribution of action on perception, perception on action, and resolving conflict. Cognitive, Affective and Behavioral Neuroscience, 17(2), 381-393. https://doi.org/10.3758/s13415-016-0485-5

Ehrsson, H. H., Kuhtz-Buschbeck, J. P., Forssberg, H., Gerloff, C., \& Hummel, F. C. (2002). Brain regions controlling nonsynergistic versus synergistic movement of the digits: a functional magnetic resonance imaging study. The Journal of Neuroscience: The Official Journal of the Society for Neuroscience, 22(12), 5074-5080. https://doi.org/22/12/5074 [pii]

Engbert, K., Wohlschläger, A., Thomas, R., \& Haggard, P. (2007). Agency, subjective time, and other minds. Journal of Experimental Psychology, 33(6), 1261-1268. https://doi.org/10.1037/0096-1523.33.6.1261

Engbert, K. Wohlschläger, A., \& Haggard, P. (2008) Who is causing what? The sense of agency 
is relational and efferent-triggered. Cognition, 107(2):693-704.

https://doi.org/10.1016/j.cognition.2007.07.021

Faul, F., Erdfelder, E., Lang, A.-G., \& Buchner, A. (2007). G*Power 3: A flexible statistical power analysis program for the social, behavioral, and biomedical sciences. Behavior Research Methods, 39(2), 175-191. https://doi.org/10.3758/BF03193146

García-Larrea, L., \& Cézanne-Bert, G. (1998). P3, Positive slow wave and working memory load: A study on the functional correlates of slow wave activity. Electroencephalography and Clinical Neurophysiology - Evoked Potentials, 108(3), 260-273. https://doi.org/10.1016/S0168-5597(97)00085-3

Gentsch, A., \& Schütz-Bosbach, S. (2011). I Did It: Unconscious Expectation of Sensory Consequences Modulates the Experience of Self-agency and Its Functional Signature. Journal of Cognitive Neuroscience, 23(12), 3817-3828. https://doi.org/10.1162/jocn_a_00012

Goldberg, M., Busch, N., \& Meer, E. Van Der. (2017). The amount of recent action-outcome coupling modulates the mechanisms of the intentional binding e ff ect : A behavioral and ERP study. Consciousness and Cognition, (August 2016), 0-1. https://doi.org/10.1016/j.concog.2017.07.001

Greenwald, a G. (1972). On doing two things at once: time sharing as a function of ideomotor compatibility. Journal of Experimental Psychology, 94(1), 52-57. https://doi.org/10.1037/h0032762

Grützmann, R., Riesel, A., Klawohn, J., Kathmann, N., \& Endrass, T. (2014). Complementary modulation of $\mathrm{N} 2$ and CRN by conflict frequency. Psychophysiology, 51(8), 761-772. https://doi.org/10.1111/psyp.12222

Haggard, P., Clark, S., \& Kalogeras, J. (2002). Voluntary action and conscious awareness. Nature Neuroscience, 5, 382-385. https://doi.org/10.1038/nn827

Haggard, P., \& Tsakiris, M. (2009). The Experience of Agency. Current Directions in Psychological Science, 18(4), 242-246. https://doi.org/10.1111/j.1467-8721.2009.01644.x

Hirai, M., Fukushima, H., \& Hiraki, K. (2003). An event-related potentials study of biological motion perception in humans. Neuroscience Letters, 344(1), 41-44. https://doi.org/10.1016/S0304-3940(03)00413-0 
Hirai, M., Senju, A., Fukushima, H., \& Hiraki, K. (2005). Active processing of biological motion perception: An ERP study. Cognitive Brain Research, 23(2-3), 387-396. https://doi.org/10.1016/j.cogbrainres.2004.11.005

Holeckova, I., Fischer, C., Giard, M. H., Delpuech, C., \& Morlet, D. (2006). Brain responses to a subject's own name uttered by a familiar voice. Brain Research, 1082(1), 142-152. https://doi.org/10.1016/j.brainres.2006.01.089

Hughes, G., Desantis, A., \& Waszak, F. (2013a). Attenuation of auditory N1 results from identity-specific action-effect prediction. European Journal of Neuroscience, 37(7), 11521158. https://doi.org/10.1111/ejn.12120

Hughes, G., Desantis, A., \& Waszak, F. (2013b). Mechanisms of intentional binding and sensory attenuation: The role of temporal prediction, temporal control, identity prediction, and motor prediction. Psychological Bulletin, 139(1), 133-151. https://doi.org/10.1037/a0028566

Huster, R. J., Westerhausen, R., Pantev, C., \& Konrad, C. (2010). The role of the cingulate cortex as neural generator of the N200 and P300 in a tactile response inhibition task. Human Brain Mapping, 31(8), 1260-1271. https://doi.org/10.1002/hbm.20933

Jo, H.-G., Wittmann, M., Hinterberger, T., \& Schmidt, S. (2014). The readiness potential reflects intentional binding. Frontiers in Human Neuroscience, 8(June), 421. https://doi.org/10.3389/fnhum.2014.00421

Kok, A. (2001). On the utility of P3 amplitude as a measure of processing capacity. Psychophysiology, 38(3), 557-577. https://doi.org/10.1017/S0048577201990559

Kornblum, S., Hasbroucq, T., \& Osman, A. (1990). Dimensional overlap: cognitive basis for stimulus-response compatibility--a model and taxonomy. Psychological Review, 97(2), 253-270. https://doi.org/10.1037/0033-295X.97.2.253

Krakowski, A. I., Ross, L. A., Snyder, A. C., Sehatpour, P., Kelly, S. P., \& Foxe, J. J. (2011). The neurophysiology of human biological motion processing: A high-density electrical mapping study. NeuroImage, 56(1), 373-383. https://doi.org/10.1016/j.neuroimage.2011.01.058

Kühn, S., Brass, M., \& Haggard, P. (2013). Feeling in control: Neural correlates of experience of agency. Cortex, 49(7), 1935-1942. https://doi.org/10.1016/j.cortex.2012.09.002 
Kühn, S., Nenchev, I., Haggard, P., Brass, M., Gallinat, J., \& Voss, M. (2011). Whodunnit? electrophysiological correlates of agency judgements. PLoS ONE, 6. https://doi.org/10.1371/journal.pone.0028657

Kwapil, T. R., Barrantes-Vidal, N., \& Silvia, P. J. (2008). The dimensional structure of the Wisconsin Schizotypy Scales: factor identification and construct validity. Schizophrenia Bulletin, 34, 444-457. https://doi.org/10.1093/schbul/sbm098

Libet, B., Gleason, C. A., Wright, E. W., \& Pearl, D. K. (1983). Time of conscious intention to act in relation to onset of cerebral activity (readiness-potential). The unconscious initiation of a freely voluntary act. Brain : A Journal of Neurology, 106 (Pt 3), 623-642. https://doi.org/10.1093/brain/106.3.623

Longo, M. R., Musil, J. J., \& Haggard, P. (2012). Visuo-tactile integration in personal space. Journal of Cognitive Neuroscience, 24(3), 543-552. https://doi.org/10.1162/jocn_a_00158

Lucci, G., Berchicci, M., Spinelli, D., \& Di Russo, F. (2014). The motor preparation of directionally incompatible movements. NeuroImage, 91, 33-42. https://doi.org/10.1016/j.neuroimage.2014.01.013

Nieuwenhuis, S., Aston-Jones, G., \& Cohen, J. D. (2005). Decision making, the P3, and the locus coeruleus--norepinephrine system. Psychological Bulletin, 131(4), 510-532. https://doi.org/10.1037/0033-2909.131.4.510

Pailing, P. E., \& Segalowitz, S. J. (2004). The effects of uncertainty in error monitoring on associated ERPs. Brain and Cognition, 56(2 SPEC. ISS.), 215-233. https://doi.org/10.1016/j.bandc.2004.06.005

Perrin, F., Pernier, J., Bertrand, O., \& Echallier, J. F. (1989). Spherical splines for scalp potential and current density mapping. Electroencephalography and Clinical Neurophysiology, 72(2), 184-187. https://doi.org/10.1016/0013-4694(89)90180-6

Polich, J. (2007). Updating P300: An integrative theory of P3a and P3b. Clinical Neurophysiology. https://doi.org/10.1016/j.clinph.2007.04.019

Poonian, S. K., Mcfadyen, J., Ogden, J., \& Cunnington, R. (2015). Implicit Agency in Observed Actions : Evidence for N1 Suppression of Tones Caused by Self-made and Observed Actions, 752-764. https://doi.org/10.1162/jocn

Preston,J. \& Wegner, D.M.(2007). The eureka error: Inadvertent plagiarism by misattributions 
of effort. Journal of Personality and Social Psychology, 92(4),575-584

Preston, J., \& Wegner, D.M.(2009). Elbow grease: The experience of effort in action. In: Morsella E, Bargh JA, Gollwitzer PM, editors. Oxford handbook of human action. Oxford University Press; New York: 2009. pp. 469-486.

RAGOT, R., \& FIORI, N. (1994). Mental processing during reactions toward and away from a stimulus: An ERP analysis of auditory congruence and S-R compatibility. Psychophysiology, 31(5), 439-446. https://doi.org/10.1111/j.1469-8986.1994.tb01047.x

Ragot, R., \& Renault, B. (1981). P300, as a function of S-R compatibility and motor programming. Biological Psychology, 13(C), 289-294. https://doi.org/10.1016/03010511(81)90044-2

Sadato, N., Yonekura, Y., Waki, A., Yamada, H., \& Ishii, Y. (1997). Role of the supplementary motor area and the right premotor cortex in the coordination of bimanual finger movements. Journal of Neuroscience, 17(24), 9667-9674. https://doi.org/10.1016/S01680102(97)90507-1

Sebanz, N., Knoblich, G., Prinz, W., \& Wascher, E. (2006). Twin peaks: An ERP study of action planning and control in coacting individuals. Journal of Cognitive Neuroscience, 18(5), 859-870. https://doi.org/10.1162/jocn.2006.18.5.859

Shibasaki, H., \& Hallett, M. (2006). What is the Bereitschaftspotential? Clinical Neurophysiology: Official Journal of the International Federation of Clinical Neurophysiology, 117(11), 2341-2356. https://doi.org/10.1016/j.clinph.2006.04.025

Sidarus, N., \& Haggard, P. (2016). Difficult action decisions reduce the sense of agency: A study using the Eriksen flanker task. Acta Psychologica, 166, 1-11. https://doi.org/10.1016/j.actpsy.2016.03.003

Sidarus, N., Vuorre, M., \& Haggard, P. (2017). How action selection influences the sense of agency: An ERP study. NeuroImage, 150(September 2016), 1-13. https://doi.org/10.1016/j.neuroimage.2017.02.015

Sidarus, N., Vuorre, M., Metcalfe, J., \& Haggard, P. (2017). Investigating the prospective sense of agency: Effects of processing fluency, stimulus ambiguity, and response conflict. Frontiers in Psychology, 8(APR). https://doi.org/10.3389/fpsyg.2017.00545

Smith, S. M., \& Nichols, T. E. (2009). Threshold-free cluster enhancement: Addressing 
problems of smoothing, threshold dependence and localisation in cluster inference.

NeuroImage, 44(1), 83-98. https://doi.org/10.1016/j.neuroimage.2008.03.061

Tecce, J. J. (1972). Contingent negative variation (CNV) and psychological processes in man. Psychological Bulletin, 77, 73-108. https://doi.org/10.1037/h0032177

Ullspererg, P., Metz, A. M., \& Gille, H. G. (1988). The P300 component of the event-related brain potential and mental effort. Ergonomics, 31(8), 1127-1137. https://doi.org/10.1080/00140138808966752

van Boxtel, G. J. M., \& Böcker, K. B. E. (2004). Cortical measures of anticipation. Journal of Psychophysiology. https://doi.org/10.1027/0269-8803.18.23.61

Vastano, R., Pozzo, T., \& Brass, M. (2017). The action congruency effect on the feelings of agency. Consciousness and Cognition, 51(December 2016), 212-222. https://doi.org/10.1016/j.concog.2017.04.002

Vastano, R., Pozzo, T., \& Brass, M. (2017). The action congruency effect on the feelings of agency. Consciousness and Cognition, 51. https://doi.org/10.1016/j.concog.2017.04.002

Vidal, F., Hasbroucq, T., Grapperon, J., \& Bonnet, M. (2000). Is the "error negativity" specific to errors? Biological Psychology, 51(2-3), 109-128. https://doi.org/10.1016/S03010511(99)00032-0

WALTER, W. G., COOPER, R., ALDRIDGE, V. J., McCALLUM, W. C., \& WINTER, A. L. (1964). Contingent Negative Variation : An Electric Sign of Sensori-Motor Association and Expectancy in the Human Brain. Nature, 203(4943), 380-384. https://doi.org/10.1038/203380a0

Waszak, F., Wascher, E., Keller, P., Koch, I., Aschersleben, G., Rosenbaum, D. A., \& Prinz, W. (2005). Intention-based and stimulus-based mechanisms in action selection. Experimental Brain Research, 162(3), 346-356. https://doi.org/10.1007/s00221-004-2183-8

Wen, W., Yamashita, A., \& Asama, H. (2015). The influence of action-outcome delay and arousal on sense of agency and the intentional binding effect. Consciousness and Cognition, 36, 87-95. https://doi.org/10.1016/j.concog.2015.06.004

Wenke, D., Fleming, S. M., \& Haggard, P. (2010). Subliminal priming of actions influences sense of control over effects of action. Cognition, 115(1), 26-38. https://doi.org/10.1016/j.cognition.2009.10.016 
White, N. C., Fawcett, J. M., \& Newman, A. J. (2014). Electrophysiological markers of biological motion and human form recognition. NeuroImage, 84, 854-867. https://doi.org/10.1016/j.neuroimage.2013.09.026

Zhang, W., \& Luck, S. J. (2009). Feature-based attention modulates feedforward visual processing. Nature Neuroscience, 12(1), 24-25. https://doi.org/10.1038/nn.2223

Zhou, B., Zhang, J. X., Li, C. A., Tan, H., \& Han, S. (2004). Spatial congruence in working memory: an ERP study. Neuroreport, 15(18), 2795-2799. 


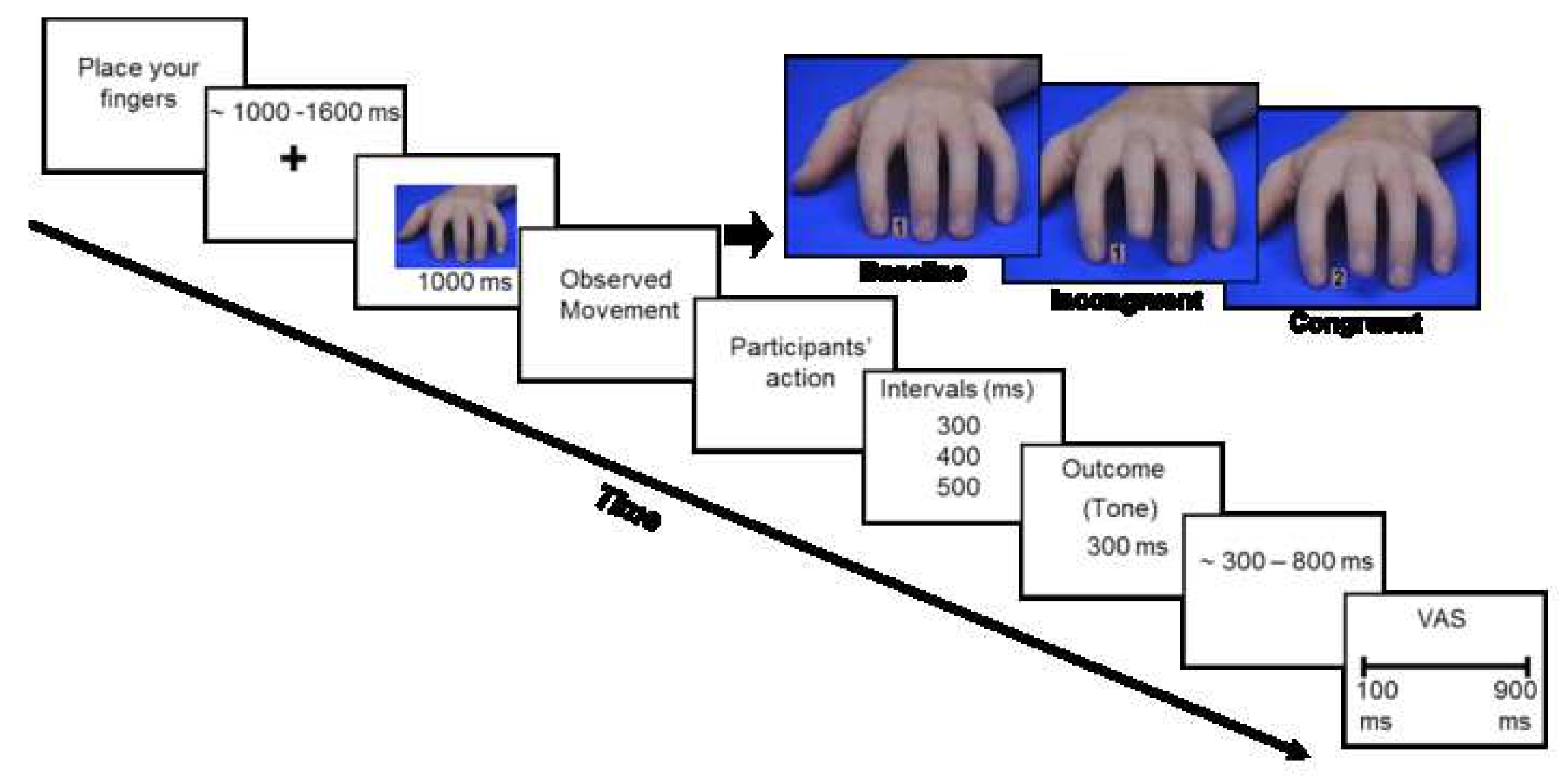

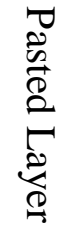

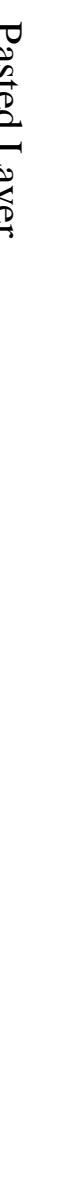




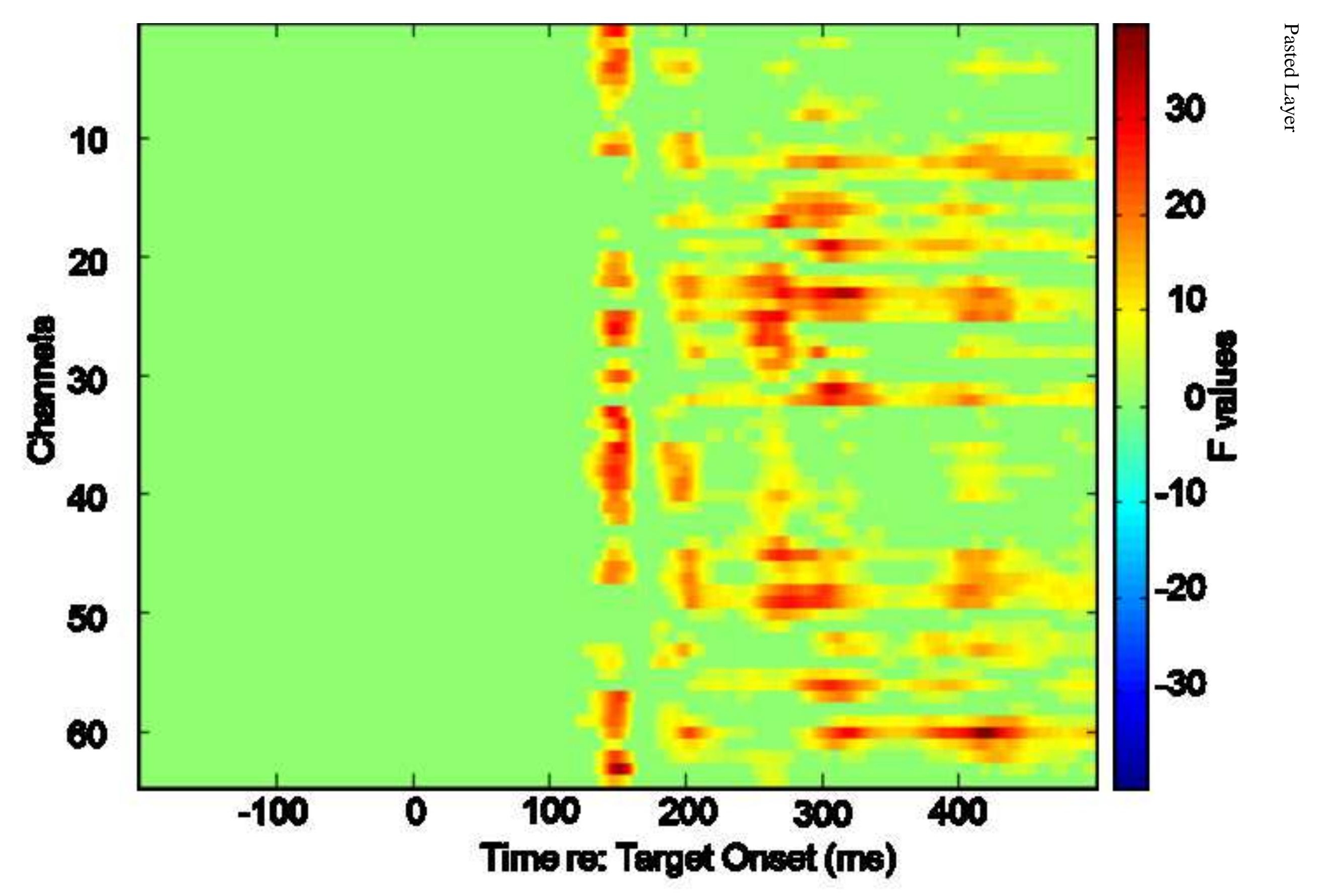

7
000
0
0
0
0
0

.



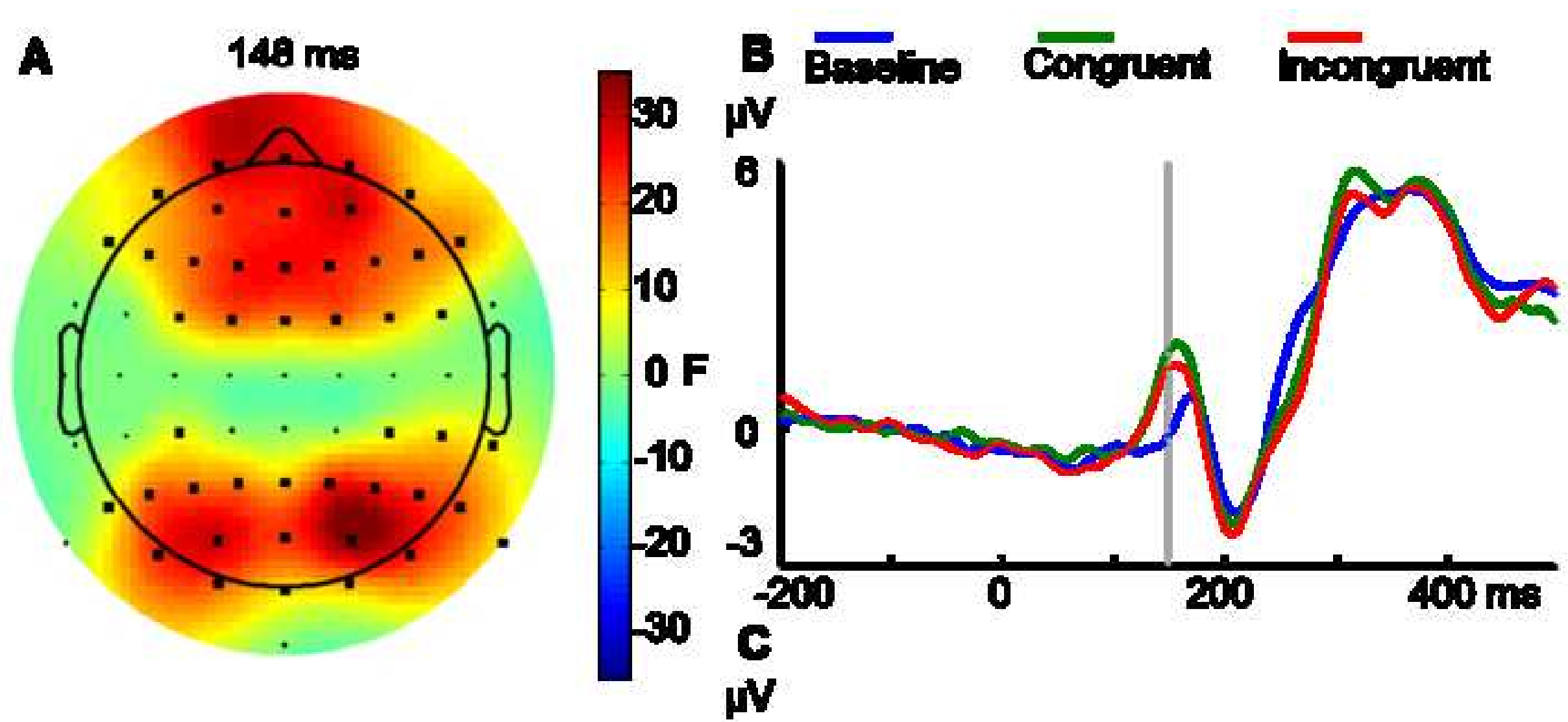

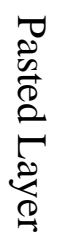

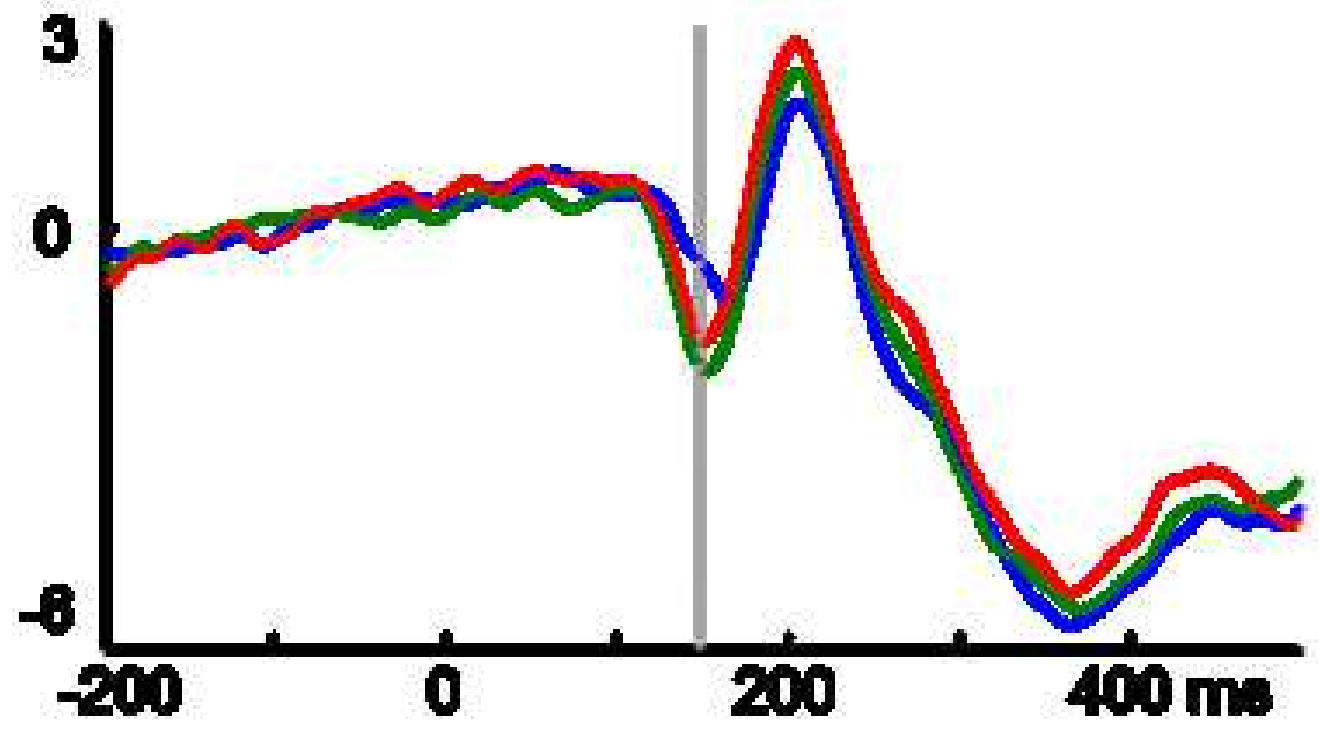



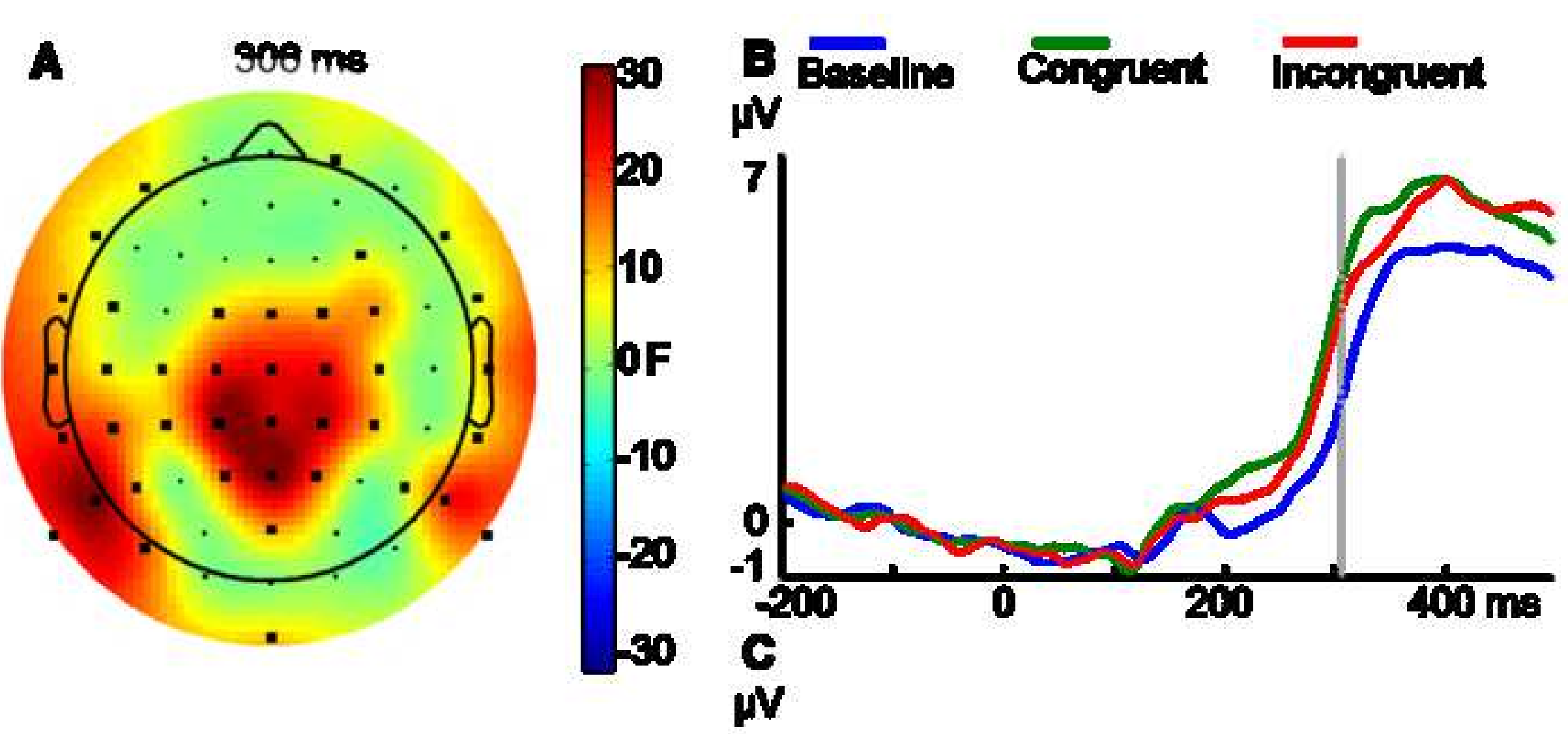

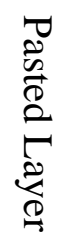

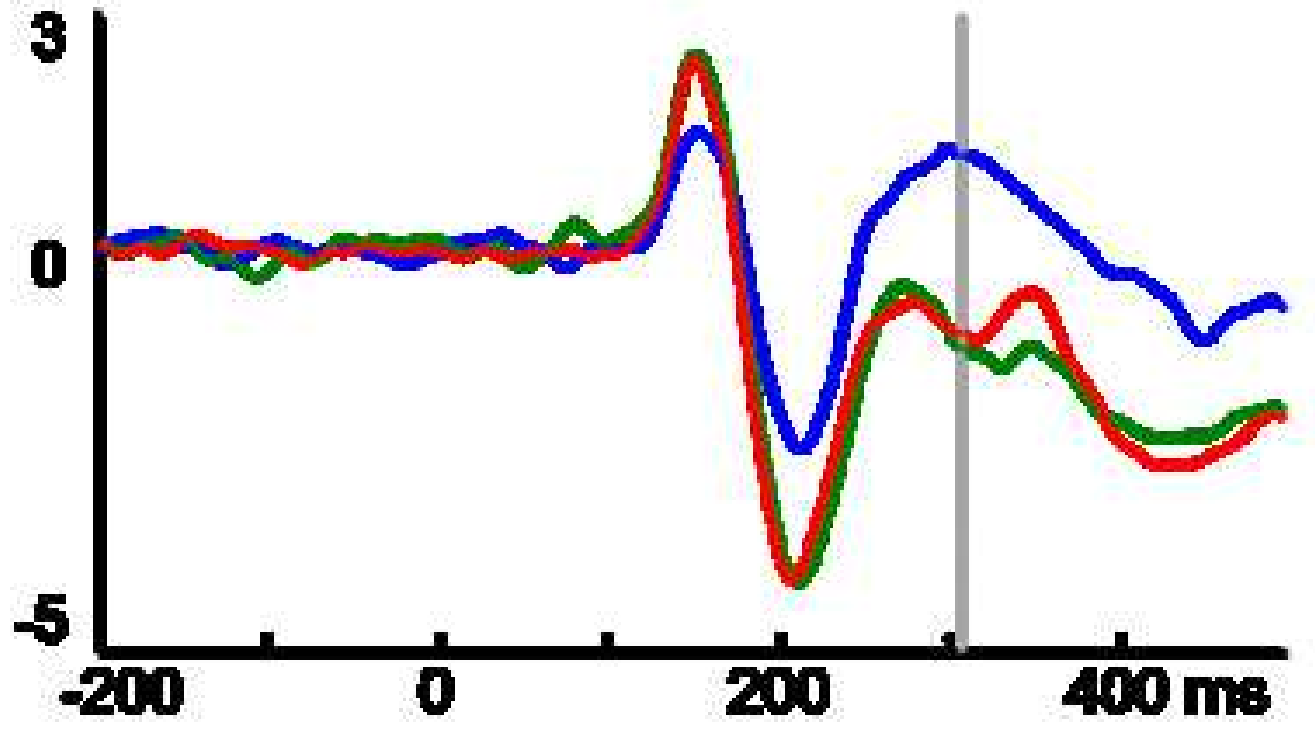




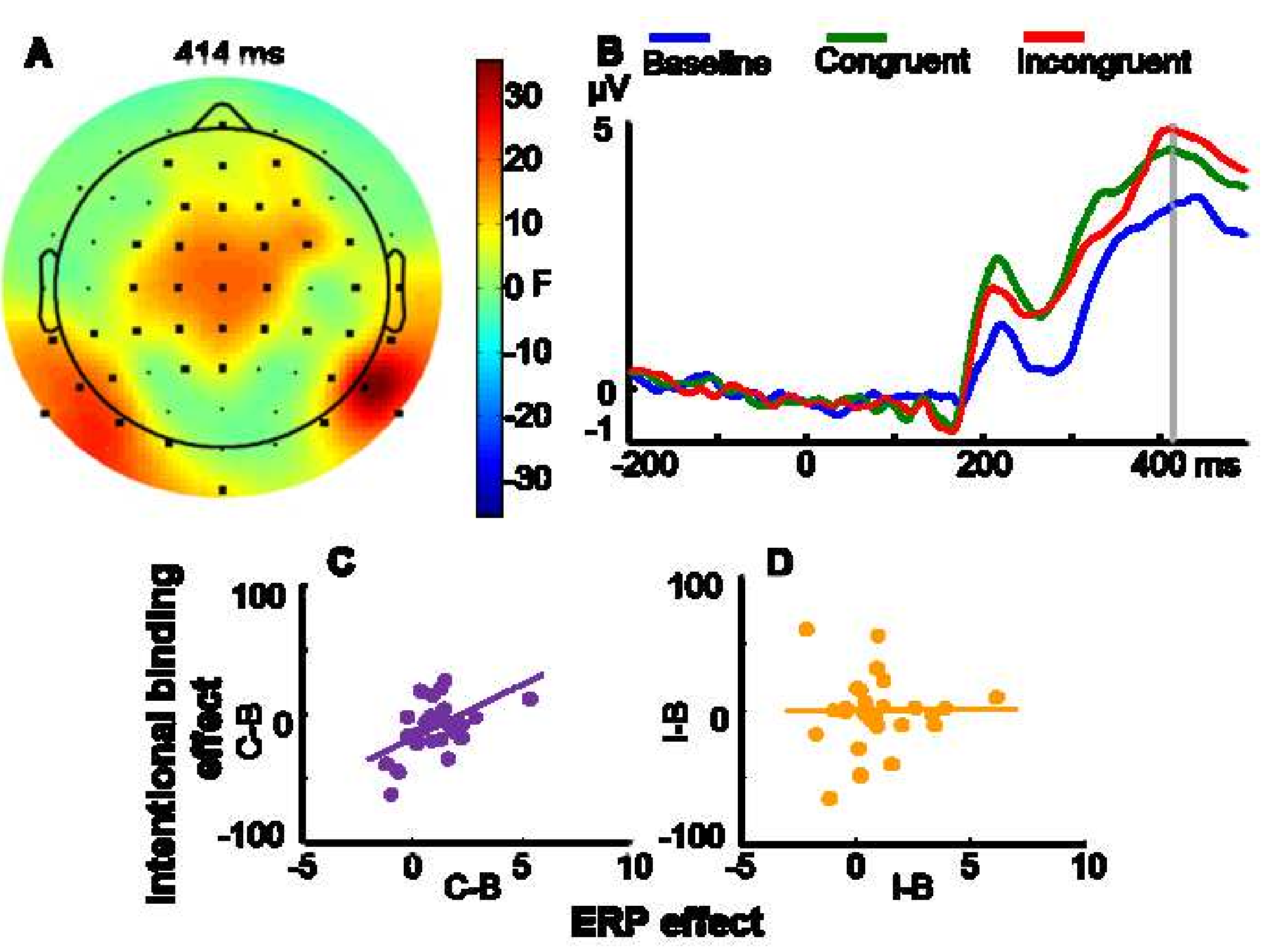


Click here to access/download;Figure;fig7.tif $\underline{\underline{\Perp}}$

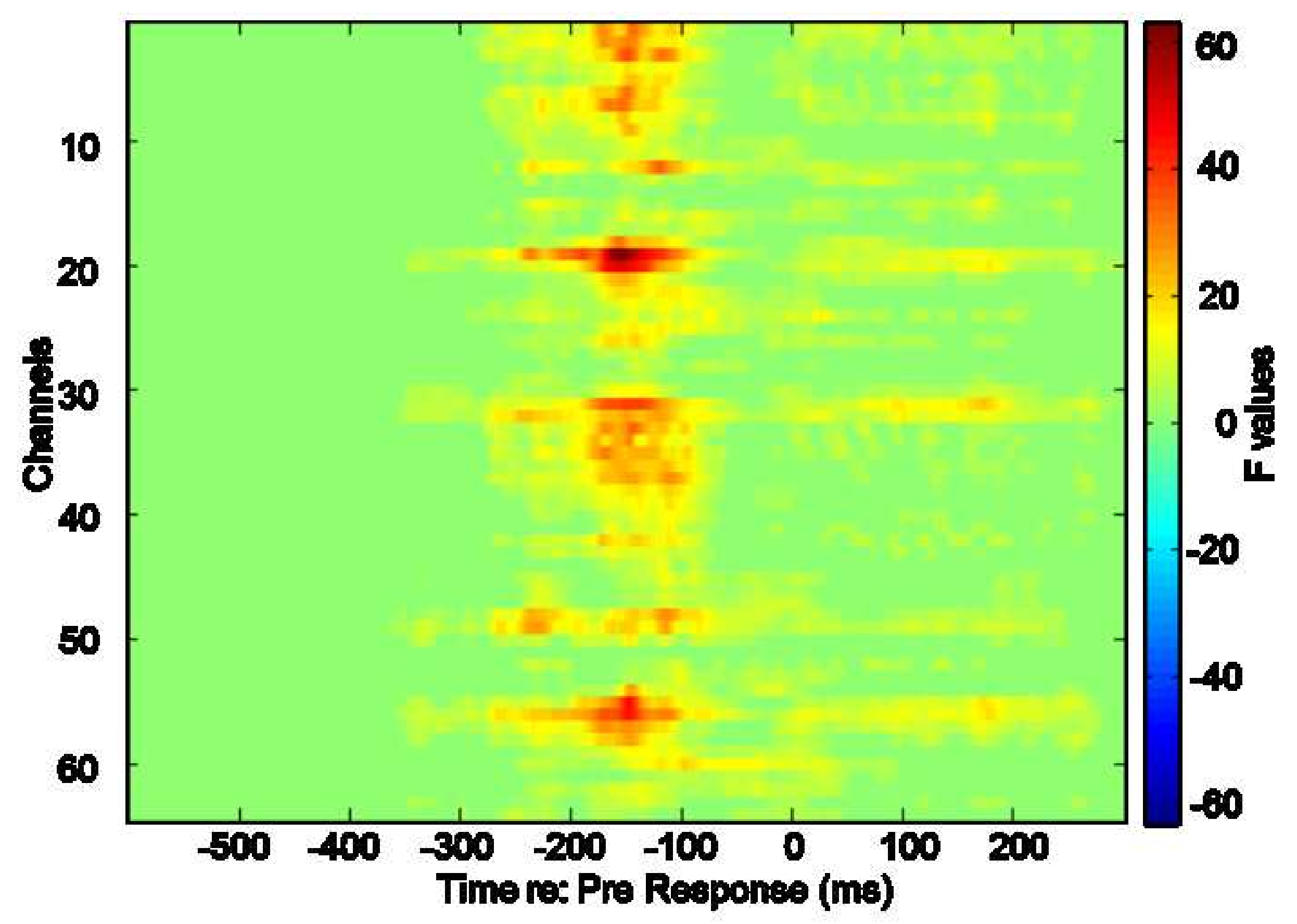

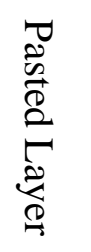



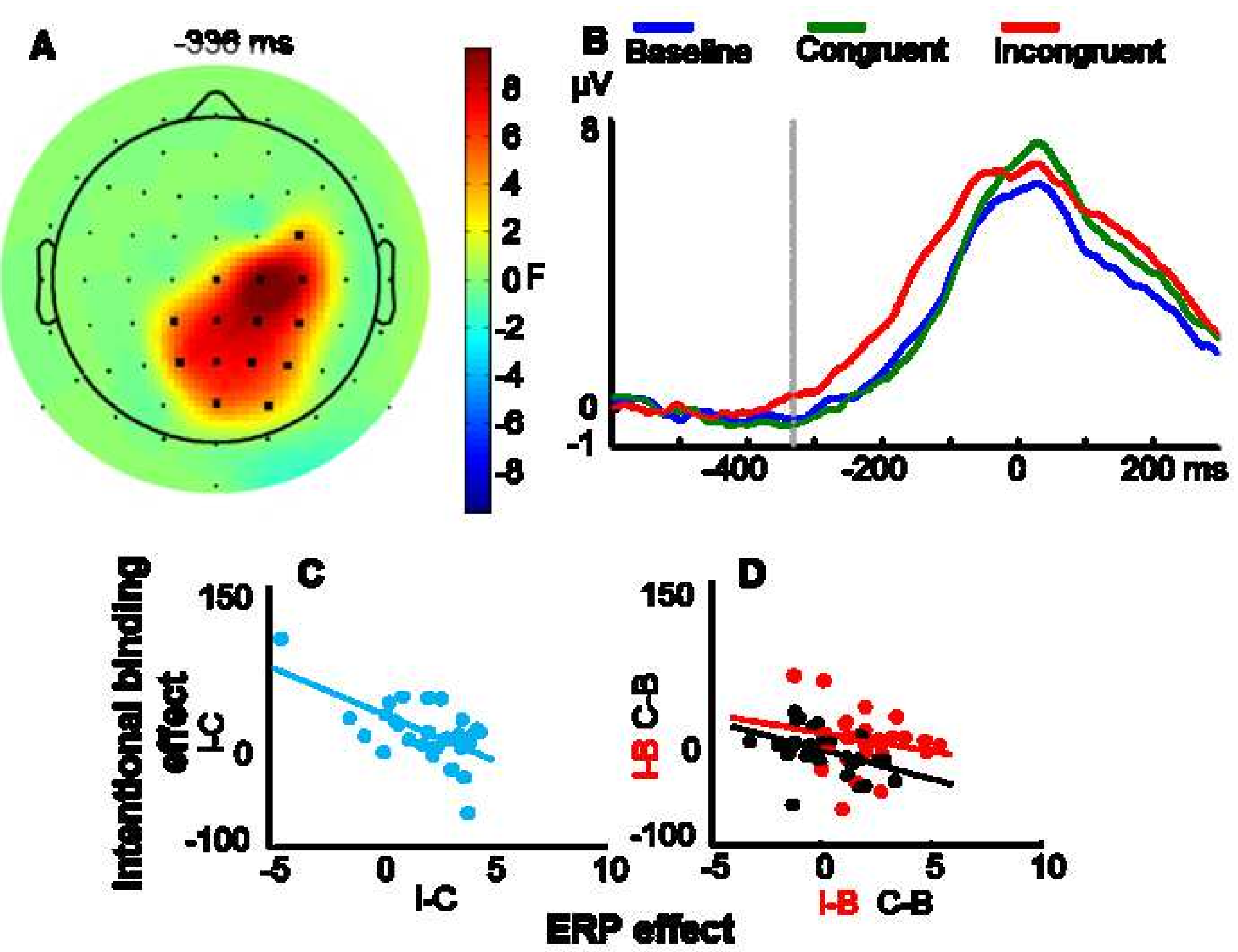

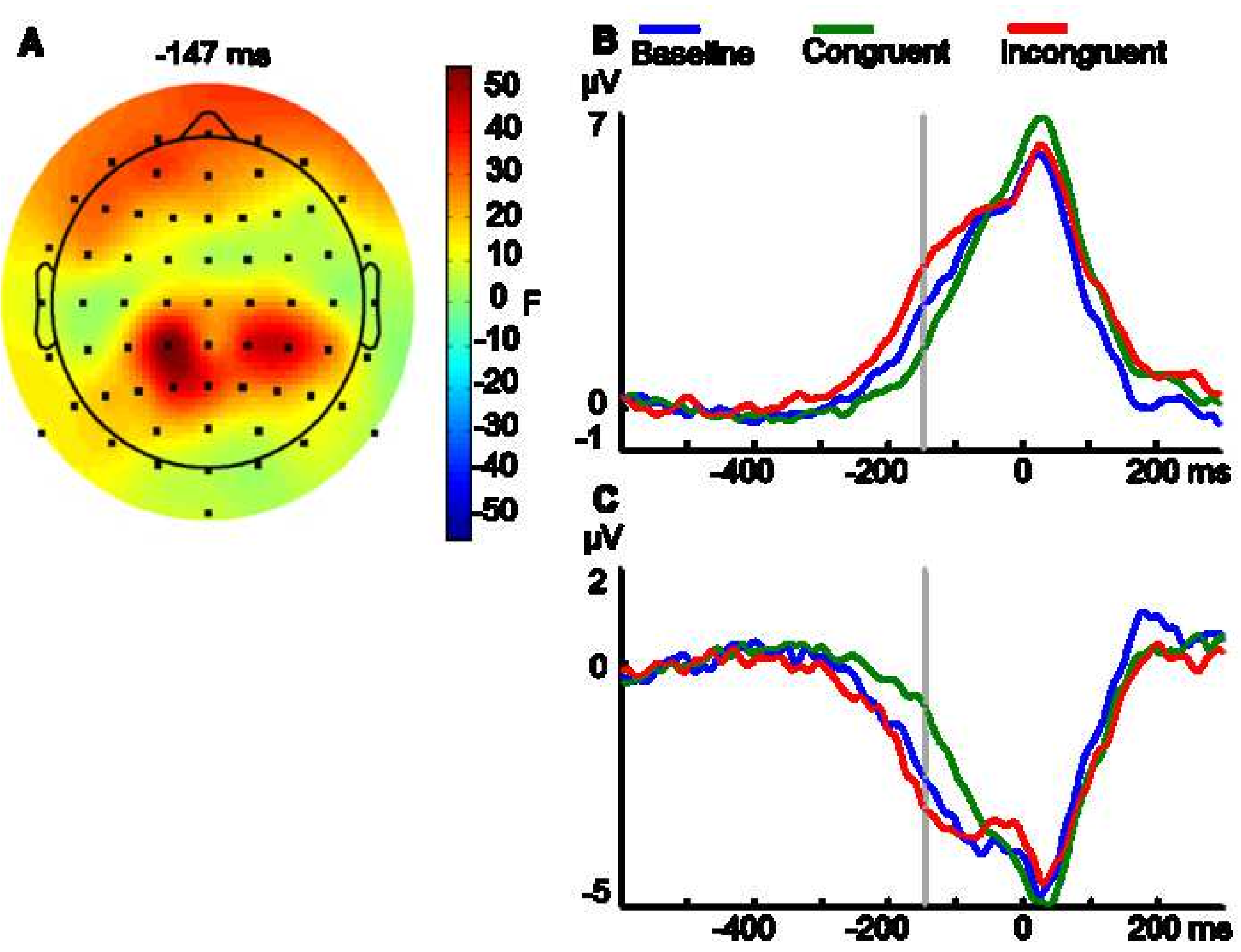


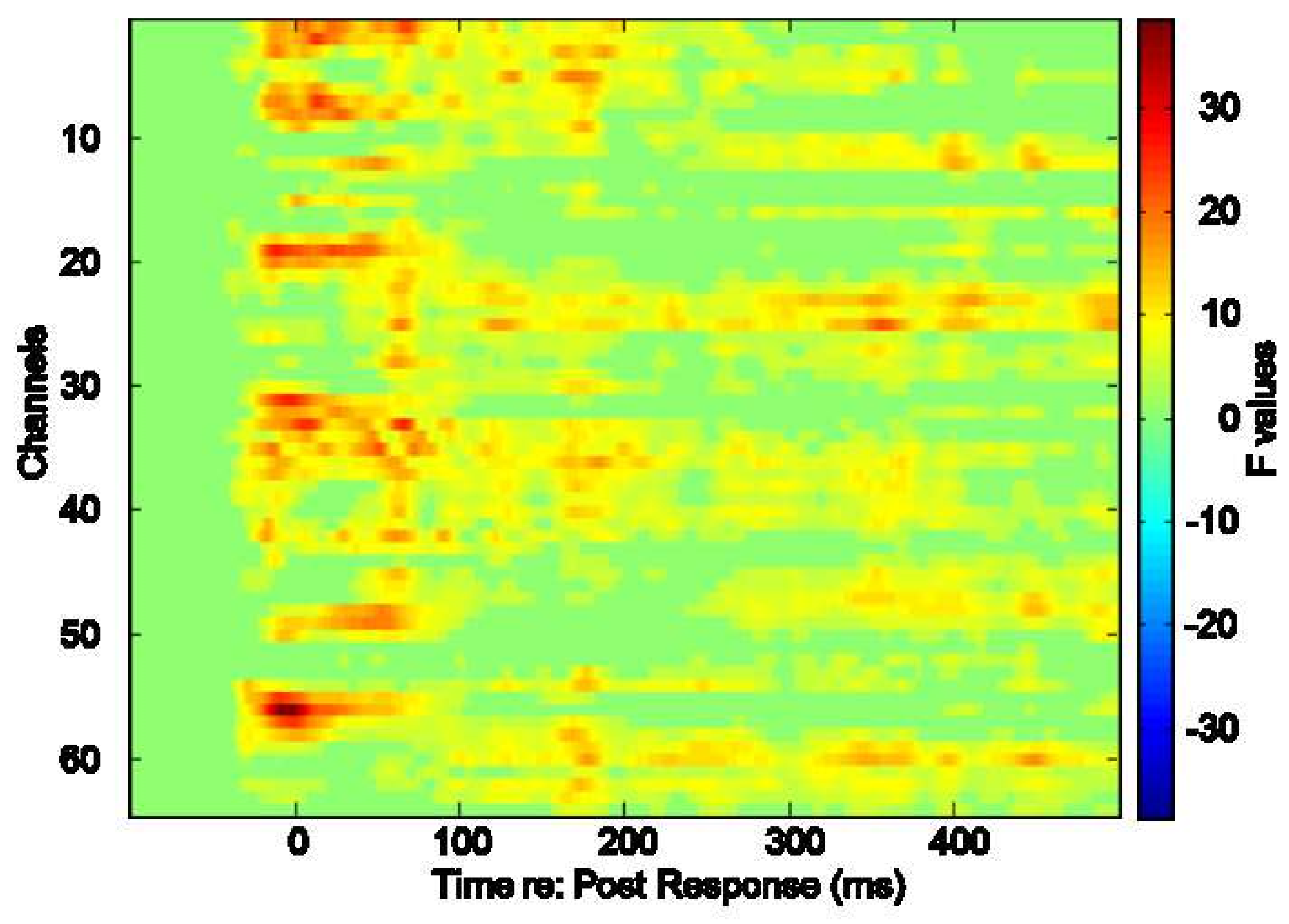



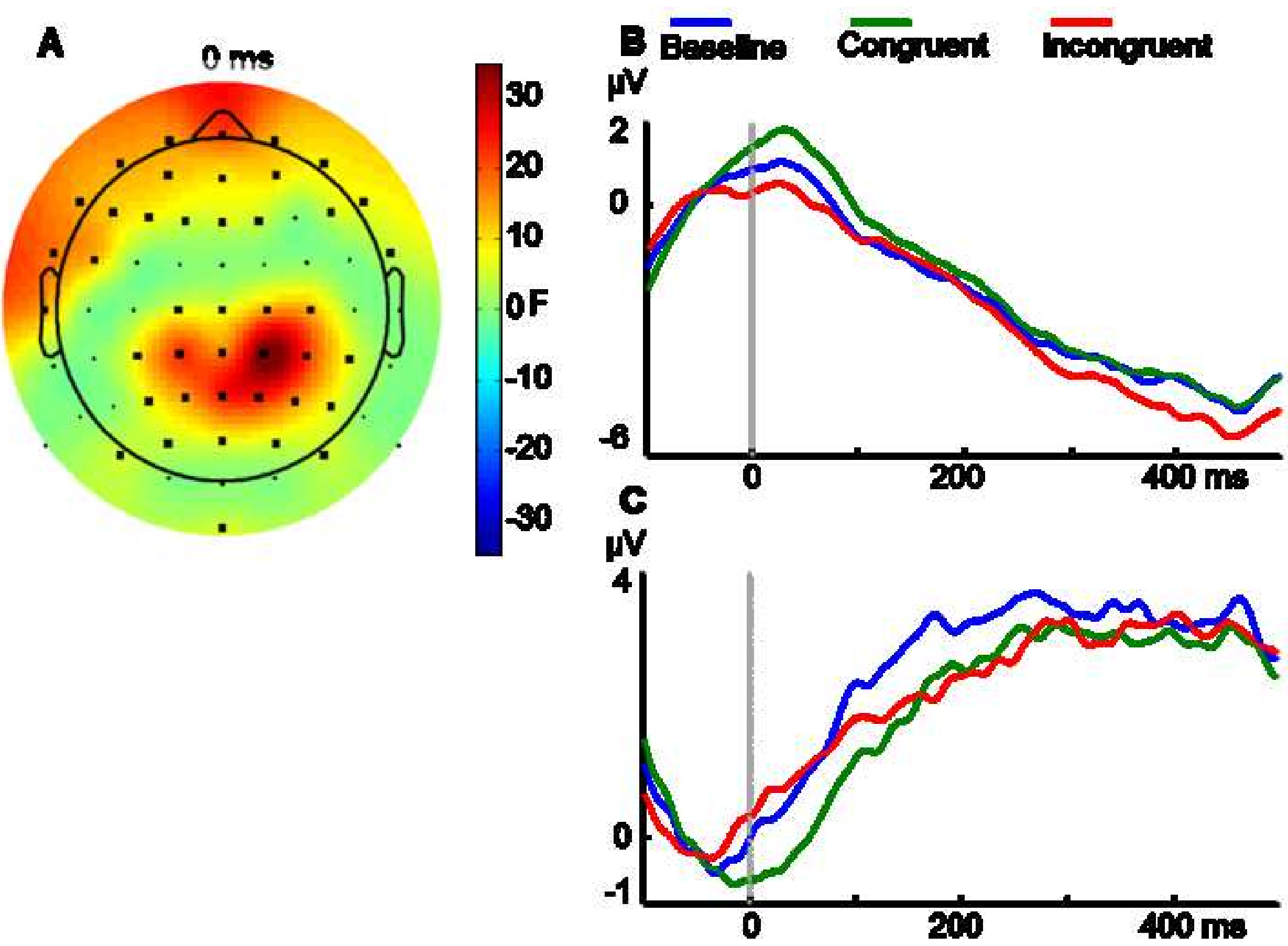

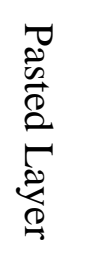



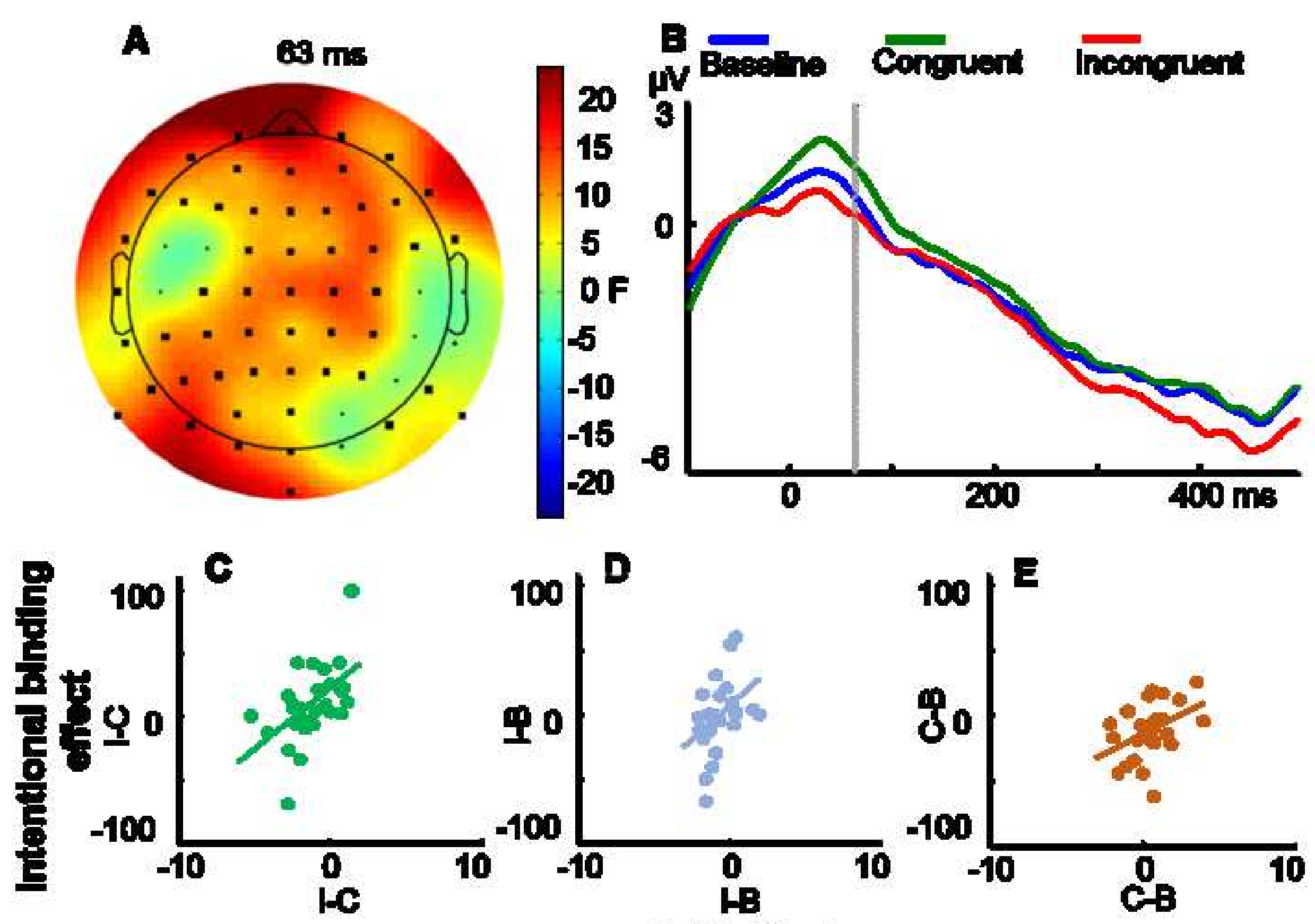

ERP eflect

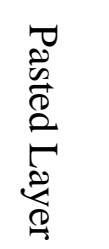

